\title{
Stationary cocycles and Busemann functions for the corner growth model
}

\author{
Nicos Georgiou' ${ }^{1}$ - Firas Rassoul-Agha ${ }^{2}$. \\ Timo Seppäläinen ${ }^{3}$
}

Received: 13 October 2015 / Revised: 18 June 2016 / Published online: 2 August 2016 (C) Springer-Verlag Berlin Heidelberg 2016

\begin{abstract}
We study the directed last-passage percolation model on the planar square lattice with nearest-neighbor steps and general i.i.d. weights on the vertices, outside of the class of exactly solvable models. Stationary cocycles are constructed for this percolation model from queueing fixed points. These cocycles serve as boundary conditions for stationary last-passage percolation, solve variational formulas that characterize limit shapes, and yield existence of Busemann functions in directions where the shape has some regularity. In a sequel to this paper the cocycles are used to prove results about semi-infinite geodesics and the competition interface.
\end{abstract}

Keywords Busemann function - Cocycle - Competition interface · Directed percolation · Geodesic · Last-passage percolation · Percolation cone · Queueing fixed point $\cdot$ Variational formula

Mathematics Subject Classification $60 \mathrm{~K} 35 \cdot 65 \mathrm{~K} 37$

Timo Seppäläinen

seppalai@math.wisc.edu

Nicos Georgiou

n.georgiou@sussex.ac.uk

Firas Rassoul-Agha

firas@math.utah.edu

1 Department of Mathematics, School of Mathematical and Physical Sciences, University of Sussex, Falmer Campus, Brighton BN1 9QH, UK

2 Mathematics Department, University of Utah, 155 South 1400 East, Salt Lake City, UT 84109, USA

3 Mathematics Department, University of Wisconsin-Madison, Van Vleck Hall, 480 Lincoln Dr., Madison, WI 53706, USA 


\section{Contents}

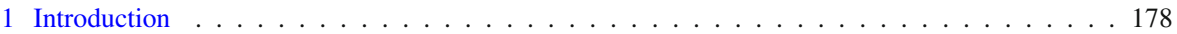

2 Preliminaries on the corner growth model . . . . . . . . . . . . . . . . . . . 181

2.1 Assumptions . . . . . . . . . . . . . . . . . . . . . . . . . . . . . . . . 182

2.2 Last-passage percolation . . . . . . . . . . . . . . . . . . . . . . . . . . . 182

2.3 Gradients and convexity . . . . . . . . . . . . . . . . . . . . . . . . . 184

2.4 Cocycles and variational formulas $\ldots \ldots \ldots \ldots \ldots$

3 Results on Busemann functions . . . . . . . . . . . . . . . . . . . . . . 186

3.1 Exactly solvable models . . . . . . . . . . . . . . . . . . . . . . . . . . . 188

3.2 Flat edge in the percolation cone . . . . . . . . . . . . . . . . 188

4 Duality . . . . . . . . . . . . . . . . . . . . . . . . 191

5 Stationary cocycles . . . . . . . . . . . . . . . . . . . . . . . . 195

5.1 Existence and properties of stationary cocycles . . . . . . . . . . . . . . . . . 195

5.2 Stationary last-passage percolation . . . . . . . . . . . . . . . . . . . . 198

5.3 Solution to the variational formulas . . . . . . . . . . . . . . . . . . . . . . . 199

6 Busemann functions from cocycles . . . . . . . . . . . . . . . . . . . . . 201

7 Cocycles from queuing fixed points . . . . . . . . . . . . . . . . . . . 209

7.1 Exactly solvable models . . . . . . . . . . . . . . . . . . . . . . . . . . . . . . . . 219

Appendix: Ergodic theorem for cocycles . . . . . . . . . . . . . . . . 220

References . . . . . . . . . . . . . . . . . . . . . . . . . 221

\section{Introduction}

We study nearest-neighbor directed last-passage percolation (LPP) on the lattice $\mathbb{Z}^{2}$, also called the corner growth model. Random i.i.d. weights $\left\{\omega_{x}\right\}_{x \in \mathbb{Z}^{2}}$ are used to define last-passage times $G_{x, y}$ between lattice points $x \leq y$ (coordinatewise ordering) in $\mathbb{Z}^{2}$ by

$$
G_{x, y}=\max _{x} \sum_{k=0}^{n-1} \omega_{x_{k}}
$$

where the maximum is over paths $x_{.}=\left\{x=x_{0}, x_{1}, \ldots, x_{n}=y\right\}$ that satisfy $x_{k+1}-$ $x_{k} \in\left\{e_{1}, e_{2}\right\}$ (up-right paths whose increments are standard basis vectors).

When $\omega_{x} \geq 0$ this defines a growth model in the first quadrant $\mathbb{Z}_{+}^{2}$. Initially the growing cluster is empty. The origin joins the cluster at time $\omega_{0}$. After both $x-e_{1}$ and $x-e_{2}$ have joined the cluster, point $x$ waits time $\omega_{x}$ to join. (However, if $x$ is on the boundary of $\mathbb{Z}_{+}^{2}$, only one of $x-e_{1}$ and $x-e_{2}$ is required to have joined.) The cluster at time $t$ is $\mathscr{C}_{t}=\left\{x \in \mathbb{Z}_{+}^{2}: G_{0, x}+\omega_{x} \leq t\right\}$. Our convention to exclude $\omega_{x_{n}}$ in (1.1) forces the addition of $\omega_{x}$ in the definition of $\mathscr{C}_{t}$.

The interest is in the large-scale behavior of the model. This begins with the deterministic limit $g_{\mathrm{pp}}(\xi)=\lim _{n \rightarrow \infty} n^{-1} G_{0,\lfloor n \xi\rfloor}$ for $\xi \in \mathbb{R}_{+}^{2}$ under a moment assumption. It is expected but not yet proved that the shape function $g_{\mathrm{pp}}$ is differentiable. Another natural expectation is that the increment process $\left\{G_{0, v_{n}+x}-G_{0, v_{n}}: x \in \mathbb{Z}^{2}\right\}$ converges in distribution as $v_{n} \rightarrow \infty$ in a particular direction. Distributionally equivalent is to look at the limit of $G_{0, v_{n}}-G_{x, v_{n}}$. For this last quantity we can expect even almost sure convergence. The limit is called a Busemann function. This is one type of result developed in the paper.

Here are some particulars of what follows, in relation to past work. 
In [27] we derived variational formulas that characterize the limiting free energy and limit shape for a general class of polymer models. The results cover both positive temperature polymers and zero temperature percolation models, both point-to-point and point-to-line models in all dimensions, and general admissible paths. Part of the theory of [27] is a solution approach to one kind of variational formula in terms of stationary cocycles.

In the present paper we construct these cocycles that minimize in the variational formula for the particular case of the planar corner growth model with general i.i.d. weights. The weights are assumed bounded from below and subject to a $2+\varepsilon$ moment bound. The construction of the cocycles comes from the fixed points of the associated queueing operator whose existence was proved by Mairesse and Prabhakar [41]. A Markov process analogy of the cocycle construction is a simultaneous construction of a given interacting Markov process for all invariant distributions, coupled through common Poisson clocks that drive the dynamics. The i.i.d. weights $\omega$ are the analogue of the clocks and the cocycles are the analogues of the initial state variables in stationary distribution.

The construction of the cocycles does not require any regularity assumptions on $g_{\mathrm{pp}}$. But the cocycles are indexed by the gradients $\nabla g_{\mathrm{pp}}(\xi)$ as $\xi$ varies across directions in the first quadrant. Consequently at a corner $\xi$ of the shape we get two cocycles that correspond to $\nabla g_{\mathrm{pp}}(\xi \pm)$. We can prove the existence of the Busemann function in directions $\xi$ where $\nabla g_{\mathrm{pp}}(\xi \pm)$ coincide and can be approximated by gradients from either side. Such directions are either (i) exposed points of differentiability of $g_{\text {pp }}$ or (ii) lie on a linear segment of $g_{\mathrm{pp}}$ whose endpoints are points of differentiability.

The companion paper [26] uses the cocycles constructed here to prove results about the geodesics and the competition interface of the corner growth model.

Under some moment and regularity assumptions on the weights, the corner growth model is expected to lie in the Kardar-Parisi-Zhang (KPZ) universality class. (For a review of KPZ universality see [18].) The fluctuations of $G_{0,\lfloor n \xi\rfloor}$ are expected to have order of magnitude $n^{1 / 3}$ and limit distributions from random matrix theory. When the weights have exponential or geometric distribution the model is exactly solvable, and it is possible to derive exact fluctuation exponents and limit distributions $[10,37,38]$. In these cases the cocycles we construct have explicit product form distributions. The present paper can be seen as an attempt to begin development of techniques for studying the corner growth model beyond the exactly solvable cases. As observed in Remark 3.6 in Sect. 3.2 below, the case of a percolation cone is exceptional as it bears some markers of weak disorder and hence cannot obey KPZ universality in all aspects.

Let us briefly touch upon the last 20 years of research on Busemann functions in percolation. The only widely applied technique for proving the existence of Busemann functions has come from the work of Newman et al. [34,39,45]. The approach uses uniqueness and coalescence of directional geodesics and relies on a uniform curvature assumption on the limit shape. This assumption cannot as yet be checked for general first or last-passage percolation models. Still there are many interesting processes with enough special features so that this approach can be carried out. The examples are exactly solvable directed lattice percolation and models in Euclidean space built on homogeneous Poisson processes. 
Wütrich [56] gave an early application of the ideas of Newman et al. to construct Busemann functions for directed last-passage percolation on Poisson points. Then came the use of Busemann functions to study competition and coexistence by Cator, Ferrari, Martin and Pimentel [15,23,24,46], existence, uniqueness and spatial mixing of invariant distributions of an interacting system by Cator and Pimentel [14], the construction of solutions to a randomly forced Burgers equation by Bakhtin, Cator, and Khanin [7,8], and Pimentel's [47] bounds on the speed of coalescence that improve those of Wütrich [56]. Distinct from this line of work is Hoffman's use of Busemann functions to study competition under more general weight distributions [31,32].

Our approach to Busemann functions (and to geodesics in the sequel [26]) is the very opposite. The limits are constructed a priori in the form of the cocycles. The cocycles define stationary percolation models that can be coupled with the original one. The coupling, ergodicity, and local regularity of the limit shape give the control that proves the Busemann limits.

The use of Busemann functions or stationary cocycles to create auxiliary stationary percolation processes has been very fruitful. Hoffman applied this idea to study geodesics and coexistence [31]. The stationary process became a tool for proving fluctuation exponents in the seminal work of Cator and Groeneboom [13] on the planar Poisson last-passage percolation model. The idea was adapted to the lattice case by Balázs et al. [10], whose estimates have then been used by other works. The stationary process of Busemann functions was also profitably utilized in [14]. In an earlier version of this technique the stationary process coupled to the percolation model was an associated particle system [53,54].

The idea of deducing existence and uniqueness of stationary processes by studying geodesic-like objects has also been used in random dynamical systems. For example, [22] and its extensions $[5,6,9,30,35]$ take this approach to produce invariant measures for the Burgers equation with random forcing. This line of work has treated situations where the space is compact or essentially compact. To make progress in the noncompact case, the approach of Newman et al. was adopted again in [7,8], as mentioned above.

The seminal results of Newman et al. on geodesics have recently been extended by Damron and Hanson [19] by taking as starting point a weak subsequential limit of Busemann functions. These weak Busemann limits of [19] can be regarded as a counterpart of our stationary cocycles.

To generalize our results beyond i.i.d. weights and potentially to higher dimensions, a possible strategy that avoids the reliance on queueing theory would be to develop sufficient control on the gradients $G_{x,\lfloor n \xi\rfloor}-G_{y,\lfloor n \xi\rfloor}$ (or their point-to-line counterparts) to construct cocycles through weak limits as $n \rightarrow \infty$. This worked well for undirected first-passage percolation in [19] because the gradients are uniformly integrable. Note however that when $\left\{\omega_{x}\right\}$ are only ergodic, the limiting shape can have corners and linear segments, and can even be a finite polygon.

Organization of the paper Section 2 introduces the corner growth model. Section 3 states the existence theorem for Busemann functions under local regularity assumptions on the limit function $g_{\mathrm{pp}}(\xi)$. The remainder of the paper does not rely on properties of $g_{\mathrm{pp}}$ beyond those known and stated in Sect. 2. Section 4 develops a 
convex duality between directions or velocities $\xi$ and tilts or external fields $h$ that comes from the relationship of the point-to-point and point-to-line percolation models. Section 5 states the existence and properties of the cocycles on which all the results of the paper are based. The proof comes at the end of the paper in Sect. 7. The cocycles are used to construct a stationary last-passage process (Sect. 5.2) and they yield minimizers for the variational formulas that characterize the limit shapes (Sect. 5.3). Section 6 proves the existence of Busemann functions.

Two particular cases of the corner growth model are addressed separately: the exactly solvable cases with geometric or exponential weights $\left\{\omega_{x}\right\}$ (Sects. 3.1, 7.1), and the percolation cone (Sect. 3.2). In the case of the percolation cone we can give an alternative formula for the limiting Busemann function and we observe that the centered Busemann function is a gradient. This is consistent with the percolation cone situation being in weak disorder, as also indicated by the vanishing of the fluctuation exponent for the point-to-line last-passage time.

We have organized the paper so that the results on Busemann functions lead and the technicalities of cocycle construction are delayed towards the end. The reader who wants to see each step fully proved before progressing further can go through the material in the following order. (i) Basic definitions from Sects. 2 and 4. (ii) The cocycle construction: Theorem 5.2 from Sect. 5.1 and its proof from Sect. 7. (iii) The stationary LPP from Sect. 5.2, followed by Theorem 6.1 for Busemann functions and its proof in Sect. 6. (iv) For minimizers of the variational formulas, Theorem 5.6 and its proof from Sect. 5.3. (v) The rest is specialization under assumptions on the weights or the limit shape.

A short Appendix states an ergodic theorem for cocycles proved in [28].

Notation and conventions $\mathbb{R}_{+}=[0, \infty), \mathbb{Z}_{+}=\{0,1,2,3, \ldots\}, \mathbb{N}=\{1,2,3, \ldots\}$. The standard basis vectors of $\mathbb{R}^{2}$ are $e_{1}=(1,0)$ and $e_{2}=(0,1)$ and the $\ell^{1}$-norm of $x \in \mathbb{R}^{2}$ is $|x|_{1}=\left|x \cdot e_{1}\right|+\left|x \cdot e_{2}\right|$. For $u, v \in \mathbb{R}^{2}$ a closed line segment on $\mathbb{R}^{2}$ is denoted by $[u, v]=\{t u+(1-t) v: t \in[0,1]\}$, and an open line segment by ]$u, v[=\{t u+(1-t) v: t \in(0,1)\}$. Coordinatewise ordering $x \leq y$ means that $x \cdot e_{i} \leq y \cdot e_{i}$ for both $i=1$ and 2. Its negation $x \not \leq y$ means that $x \cdot e_{1}>y \cdot e_{1}$ or $x \cdot e_{2}>y \cdot e_{2}$. An admissible or up-right path $x_{0, n}=\left(x_{k}\right)_{k=0}^{n}$ on $\mathbb{Z}^{2}$ satisfies $x_{k}-x_{k-1} \in\left\{e_{1}, e_{2}\right\}$.

The environment space is $\Omega=\mathbb{R}^{\mathbb{Z}^{2}}$ whose elements are denoted by $\omega$. Elements of a larger product space $\widehat{\Omega}=\Omega \times \Omega^{\prime}$ are denoted by $\widehat{\omega}=\left(\omega, \omega^{\prime}\right)$. Parameter $p>2$ appears in a moment hypothesis $\mathbb{E}\left[\left|\omega_{0}\right|^{p}\right]<\infty$, while $p_{1}$ is the probability of an open site in an oriented site percolation process. $X \sim \mu$ means that random variable $X$ has distribution $\mu$.

$\mathscr{D}$ is the set of points of differentiability of $g_{\text {pp }}$ and $\mathscr{E}$ the set of exposed points of differentiability. A statement that contains \pm or $\mp$ is a combination of two statements: one for the top choice of the sign and another one for the bottom choice.

\section{Preliminaries on the corner growth model}

This section presents assumptions, definitions, and notation used throughout the paper, and states some known results. 


\subsection{Assumptions}

The two-dimensional corner growth model is the last-passage percolation model on the planar square lattice $\mathbb{Z}^{2}$ with admissible steps $\mathscr{R}=\left\{e_{1}, e_{2}\right\} . \Omega=\mathbb{R}^{\mathbb{Z}^{2}}$ is the space of environments or weight configurations $\omega=\left(\omega_{x}\right)_{x \in \mathbb{Z}^{2}}$. The group of spatial translations $\left\{T_{x}\right\}_{x \in \mathbb{Z}^{2}}$ acts on $\Omega$ by $\left(T_{x} \omega\right)_{y}=\omega_{x+y}$ for $x, y \in \mathbb{Z}^{2}$. Let $\mathfrak{S}$ denote the Borel $\sigma$-algebra of $\Omega$. P is a Borel probability measure on $\Omega$ under which the weights $\left\{\omega_{x}\right\}$ are independent, identically distributed (i.i.d.) nondegenerate random variables with a $2+\varepsilon$ moment. Expectation under $\mathbb{P}$ is denoted by $\mathbb{E}$. For a technical reason we also assume $\mathbb{P}\left(\omega_{0} \geq c\right)=1$ for some finite constant $c$.

For future reference we summarize our standing assumptions in this statement:

$\mathbb{P}$ is i.i.d., $\mathbb{E}\left[\left|\omega_{0}\right|^{p}\right]<\infty$ for some $p>2, \sigma^{2}=\operatorname{Var}\left(\omega_{0}\right)>0$, and $\mathbb{P}\left(\omega_{0} \geq c\right)=1$ for some $c>-\infty$.

Assumption (2.1) is valid throughout the paper and will not be repeated in every statement. The constant

$$
m_{0}=\mathbb{E}\left(\omega_{0}\right)
$$

will appear frequently. The symbol $\omega$ is reserved for these $\mathbb{P}$-distributed i.i.d. weights, also later when they are embedded in a larger configuration $\widehat{\omega}=\left(\omega, \omega^{\prime}\right)$.

Assumption $\mathbb{P}\left(\omega_{0} \geq c\right)=1$ is used explicitly only in Sect. 7 where we rely on queueing theory. In that context $\omega_{x}$ is a service time, and the results we quote have been proved only for $\omega_{x} \geq 0$. (The extension to $\omega_{x} \geq c$ is immediate.) The key point is that if the queueing results are extended to general real-valued i.i.d. weights $\omega_{x}$ subject to the moment assumption in (2.1), everything in this paper is true for these general real-valued weights.

\subsection{Last-passage percolation}

Given an environment $\omega$ and two points $x, y \in \mathbb{Z}^{2}$ with $x \leq y$ coordinatewise, define the point-to-point last-passage time by

$$
G_{x, y}=\max _{x_{0, n}} \sum_{k=0}^{n-1} \omega_{x_{k}} .
$$

The maximum is over paths $x_{0, n}=\left(x_{k}\right)_{k=0}^{n}$ that start at $x_{0}=x$, end at $x_{n}=y$ with $n=|y-x|_{1}$, and have increments $x_{k+1}-x_{k} \in\left\{e_{1}, e_{2}\right\}$. Call such paths admissible or up-right.

Given a vector $h \in \mathbb{R}^{2}$, an environment $\omega$, and an integer $n \geq 0$, define the $n$-step point-to-line last-passage time with tilt (or external field) $h$ by

$$
G_{n}(h)=\max _{x_{0, n}}\left\{\sum_{k=0}^{n-1} \omega_{x_{k}}+h \cdot x_{n}\right\} .
$$


The maximum is over all admissible $n$-step paths that start at $x_{0}=0$.

It is standard (see for example [43] or [49]) that under assumption (2.1), for $\mathbb{P}$ almost every $\omega$, simultaneously for every $\xi \in \mathbb{R}_{+}^{2}$ and every $h \in \mathbb{R}^{2}$, the following limits exist:

$$
\begin{aligned}
& g_{\mathrm{pp}}(\xi)=\lim _{n \rightarrow \infty} n^{-1} G_{0,\lfloor n \xi\rfloor}, \\
& g_{\mathrm{pl}}(h)=\lim _{n \rightarrow \infty} n^{-1} G_{n}(h) .
\end{aligned}
$$

In the definition above integer parts are taken coordinatewise: $\lfloor v\rfloor=(\lfloor a\rfloor,\lfloor b\rfloor) \in \mathbb{Z}^{2}$ for $v=(a, b) \in \mathbb{R}^{2}$. A stronger result is also true: the shape theorem gives a uniform limit (Theorem 5.1(i) of [43]):

$$
\lim _{n \rightarrow \infty} n^{-1} \max _{x \in \mathbb{Z}_{2}^{+}:|x|_{1}=n}\left|G_{0, x}-g_{\mathrm{pp}}(x)\right|=0 \quad \mathbb{P} \text {-almost surely. }
$$

Under assumption (2.1) $g_{\mathrm{pp}}$ and $g_{\mathrm{pl}}$ are finite nonrandom continuous functions. In particular, $g_{\mathrm{pp}}$ is continuous up to the boundary of $\mathbb{R}_{+}^{2}$. Furthermore, $g_{\mathrm{pp}}$ is a symmetric, concave, 1-homogeneous function on $\mathbb{R}_{+}^{2}$ and $g_{\mathrm{pl}}$ is a convex Lipschitz function on $\mathbb{R}^{2}$. Homogeneity means that $g_{\mathrm{pp}}(c \xi)=c g_{\mathrm{pp}}(\xi)$ for $\xi \in \mathbb{R}_{+}^{2}$ and $c \in \mathbb{R}_{+}$. By homogeneity, for most purposes it suffices to consider $g_{\mathrm{pp}}$ as a function on the convex hull $\mathscr{U}=\left[e_{1}, e_{2}\right]=\left\{t e_{1}+(1-t) e_{2}: 0 \leq t \leq 1\right\}$ of $\mathscr{R}$. The relative interior ri $\mathscr{U}$ is the open line segment $] e_{1}, e_{2}\left[=\left\{t e_{1}+(1-t) e_{2}: 0<t<1\right\}\right.$.

Decomposing according to the endpoint of the path and some estimation (Theorem 2.2 in [49]) give

$$
g_{\mathrm{pl}}(h)=\sup _{\xi \in \mathscr{U}}\left\{g_{\mathrm{pp}}(\xi)+h \cdot \xi\right\} .
$$

By convex duality for $\xi \in$ ri $\mathscr{U}$

$$
g_{\mathrm{pp}}(\xi)=\inf _{h \in \mathbb{R}^{2}}\left\{g_{\mathrm{pl}}(h)-h \cdot \xi\right\}
$$

Let us say $\xi \in \operatorname{ri} \mathscr{U}$ and $h \in \mathbb{R}^{2}$ are dual if

$$
g_{\mathrm{pp}}(\xi)=g_{\mathrm{pl}}(h)-h \cdot \xi
$$

Very little is known in general about $g_{\text {pp }}$ beyond the soft properties mentioned above. In the exactly solvable case, with $\omega_{x}$ either exponential or geometric, we have $g_{\mathrm{pp}}(s, t)=(s+t) m_{0}+2 \sigma \sqrt{s t}$. The Durrett-Liggett flat edge result ([21], Theorem 3.3 below) tells us that this formula is not true for all i.i.d. weights. It does hold for general weights asymptotically at the boundary [43]: $g_{\mathrm{pp}}(1, t)=m_{0}+2 \sigma \sqrt{t}+o(\sqrt{t})$ as $t \searrow 0$. 


\subsection{Gradients and convexity}

Regularity properties of $g_{\text {pp }}$ play a role in our results, so we introduce notation for that purpose. Let

$$
\mathscr{D}=\left\{\xi \in \text { ri } \mathscr{U}: g_{\text {pp }} \text { is differentiable at } \xi\right\} .
$$

To be clear, $\xi \in \mathscr{D}$ means that the gradient $\nabla g_{\mathrm{pp}}(\xi)$ exists in the usual sense of differentiability of functions of several variables. At $\xi \in \mathrm{ri} \mathscr{U}$ this is equivalent to the differentiability of the single variable function $r \mapsto g_{\mathrm{pp}}(r, 1-r)$ at $r=\xi \cdot e_{1} /|\xi|_{1}$. By concavity the set (ri $\mathscr{U}) \backslash \mathscr{D}$ is at most countable.

A point $\xi \in$ ri $\mathscr{U}$ is an exposed point if

$$
\exists v \in \mathbb{R}^{2} \text { such that } \forall \zeta \in \operatorname{ri} \mathscr{U} \backslash\{\xi\}: g_{\mathrm{pp}}(\zeta)<g_{\mathrm{pp}}(\xi)+v \cdot(\zeta-\xi)
$$

The set of exposed points of differentiability of $g_{\text {pp }}$ is $\mathscr{E}=\{\xi \in \mathscr{D}:(2.8)$ holds $\}$. For $\xi \in \mathscr{E},(2.8)$ is uniquely satisfied by $v=\nabla g_{\mathrm{pp}}(\xi)$. The condition for an exposed point is formulated entirely in terms of $\mathscr{U}$ because $g_{\text {pp }}$ is a homogeneous function and therefore cannot have exposed points as a function on $\mathbb{R}_{+}^{2}$.

It is expected that $g_{\mathrm{pp}}$ is differentiable on all of ri $\mathscr{U}$. But since this is not known, our development must handle possible points of nondifferentiability. For this purpose we take left and right limits on $\mathscr{U}$. Our convention is that a left limit $\xi \rightarrow \zeta$ on $\mathscr{U}$ means that $\xi \cdot e_{1}$ increases to $\zeta \cdot e_{1}$, while in a right limit $\xi \cdot e_{1}$ decreases to $\zeta \cdot e_{1}$, with $\xi \neq \zeta$.

For $\zeta \in$ ri $\mathscr{U}$ define one-sided gradient vectors $\nabla g_{\mathrm{pp}}(\zeta \pm)$ by

$$
\begin{aligned}
\nabla g_{\mathrm{pp}}(\zeta \pm) \cdot e_{1} & =\lim _{\varepsilon \searrow 0} \frac{g_{\mathrm{pp}}\left(\zeta \pm \varepsilon e_{1}\right)-g_{\mathrm{pp}}(\zeta)}{ \pm \varepsilon} \\
\text { and } \nabla g_{\mathrm{pp}}(\zeta \pm) \cdot e_{2} & =\lim _{\varepsilon \searrow 0} \frac{g_{\mathrm{pp}}\left(\zeta \mp \varepsilon e_{2}\right)-g_{\mathrm{pp}}(\zeta)}{\mp \varepsilon} \text {. }
\end{aligned}
$$

Concavity of $g_{\mathrm{pp}}$ ensures the limits exist. $\nabla g_{\mathrm{pp}}(\xi \pm)$ coincide (and equal $\nabla g_{\mathrm{pp}}(\xi)$ ) if and only if $\xi \in \mathscr{D}$. Furthermore, on ri $\mathscr{U}$,

$$
\nabla g_{\mathrm{pp}}(\zeta-)=\lim _{\xi \cdot e_{1} \nearrow \zeta \cdot e_{1}} \nabla g_{\mathrm{pp}}(\xi \pm) \quad \text { and } \nabla g_{\mathrm{pp}}(\zeta+)=\lim _{\xi \cdot e_{1} \searrow \zeta \cdot e_{1}} \nabla g_{\mathrm{pp}}(\xi \pm)
$$

For $\xi \in$ ri $\mathscr{U}$ define maximal line segments on which $g_{\mathrm{pp}}$ is linear, $\mathscr{U} \xi$ - for the left gradient at $\xi$ and $\mathscr{U} \xi+$ for the right gradient at $\xi$, by

$$
\mathscr{U}_{\xi \pm}=\left\{\zeta \in \operatorname{ri} \mathscr{U}: g_{\mathrm{pp}}(\zeta)-g_{\mathrm{pp}}(\xi)=\nabla g(\xi \pm) \cdot(\zeta-\xi)\right\}
$$

Either or both segments can degenerate to a point. Let

$$
\mathscr{U}_{\xi}=\mathscr{U} \xi-\cup \mathscr{U} \xi+=[\underline{\xi}, \bar{\xi}] \quad \text { with } \underline{\xi} \cdot e_{1} \leq \bar{\xi} \cdot e_{1} \text {. }
$$




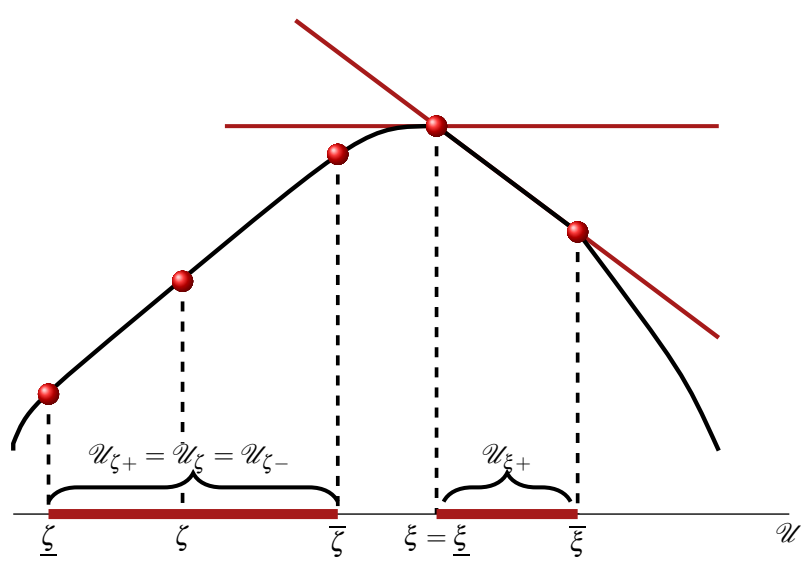

Fig. 1 A graph of a concave function over $\mathscr{U}$ to illustrate the definitions. $\underline{\zeta}, \zeta$ and $\bar{\zeta}$ are points of differentia-

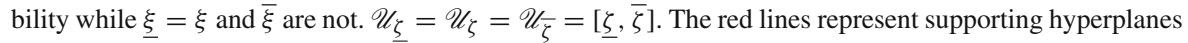
at $\xi$. The slope from the left at $\xi$ is zero, and the horizontal red line touches the graph only at $\xi$. Hence $\mathscr{U} \xi_{-}=\{\xi\}$. Points on the line segments $[\underline{\zeta}, \bar{\zeta}]$ and $] \xi, \bar{\xi}[$ are not exposed. $\mathscr{E}=$ ri $\mathscr{U} \backslash([\zeta, \bar{\zeta}] \cup[\xi, \bar{\xi}])$

If $\xi \in \mathscr{D}$ then $\mathscr{U}_{\xi+}=\mathscr{U}_{\xi-}=\mathscr{U}_{\xi}$, while if $\xi \notin \mathscr{D}$ then $\mathscr{U}_{\xi+} \cap \mathscr{U} \xi-=\{\xi\}$. If $\xi \in \mathscr{E}$ then $\mathscr{U}_{\xi}=\{\xi\}$. Figure 1 illustrates.

For $\zeta \cdot e_{1}<\eta \cdot e_{1}$ in ri $\mathscr{U},[\zeta, \eta]$ is a maximal linear segment of $g_{\mathrm{pp}}$ if $\nabla g_{\mathrm{pp}}$ exists and is constant in $\zeta, \eta$ [ but not on any strictly larger open line segment in ri $\mathscr{U}$. Then $[\zeta, \eta]=\mathscr{U}_{\zeta+}=\mathscr{U}_{\eta-}=\mathscr{U}_{\xi}$ for any $\left.\xi \in\right] \zeta, \eta\left[\right.$. If $\zeta, \eta \in \mathscr{D}$ we say that $g_{\text {pp }}$ is differentiable at the endpoints of this maximal linear segment. This hypothesis will be invoked several times.

\subsection{Cocycles and variational formulas}

The next definition is central to the paper.

Definition 2.1 (Cocycle) A measurable function $B: \Omega \times \mathbb{Z}^{2} \times \mathbb{Z}^{2} \rightarrow \mathbb{R}$ is a stationary $L^{1}(\mathbb{P})$ cocycle if it satisfies the following three conditions.

(a) Integrability: for each $z \in\left\{e_{1}, e_{2}\right\}, \mathbb{E}|B(0, z)|<\infty$.

(b) Stationarity: for $\mathbb{P}$-a.e. $\omega$ and all $x, y, z \in \mathbb{Z}^{2}, B(\omega, z+x, z+y)=B\left(T_{z} \omega, x, y\right)$.

(c) Additivity: for $\mathbb{P}$-a.e. $\omega$ and all $x, y, z \in \mathbb{Z}^{2}, B(\omega, x, y)+B(\omega, y, z)=B(\omega, x, z)$.

The space of stationary $L^{1}(\mathbb{P})$ cocycles on $(\Omega, \mathfrak{S}, \mathbb{P})$ is denoted by $\mathscr{K}(\Omega)$. The subspace $\mathscr{K}_{0}(\Omega)$ of centered stationary $L^{1}(\mathbb{P})$ cocycles consists of $F \in \mathscr{K}(\Omega)$ such that $\mathbb{E}[F(x, y)]=0$ for all $x, y \in \mathbb{Z}^{2}$.

$\mathscr{K}_{0}(\Omega)$ is the $L^{1}(\mathbb{P})$ closure of gradients $F(\omega, x, y)=\varphi\left(T_{y} \omega\right)-\varphi\left(T_{x} \omega\right), \varphi \in L^{1}(\mathbb{P})$ (see [50, Lemma C.3]). Our convention for centering a stationary $L^{1}$ cocycle $B$ is to let $h(B) \in \mathbb{R}^{2}$ denote the vector that satisfies

$$
\mathbb{E}\left[B\left(0, e_{i}\right)\right]=-h(B) \cdot e_{i} \quad \text { for } i \in\{1,2\}
$$


and then define $F \in \mathscr{K}_{0}(\Omega)$ by

$$
F(\omega, x, y)=h(B) \cdot(x-y)-B(\omega, x, y) .
$$

Cocycles appear as minimizers in variational formulas that describe the limits of last-passage percolation models. In Theorems 3.2 and 4.3 in [27] we proved these variational formulas: for $h \in \mathbb{R}^{2}$

$$
g_{\mathrm{pl}}(h)=\inf _{F \in \mathscr{K}_{0}(\Omega)} \mathbb{P} \text {-ess sup } \max _{i \in\{1,2\}}\left\{\omega_{0}+h \cdot e_{i}+F\left(\omega, 0, e_{i}\right)\right\}
$$

and for $\xi \in$ ri $\mathscr{U}$

$$
g_{\text {pp }}(\xi)=\inf _{B \in \mathscr{K}(\Omega)} \mathbb{P} \text {-ess sup } \max _{i \in\{1,2\}}\left\{\omega_{0}-B\left(\omega, 0, e_{i}\right)-h(B) \cdot \xi\right\}
$$

\section{Results on Busemann functions}

This section utilizes specialized assumptions either on the shape function $g_{\mathrm{pp}}$ or on the weights, in addition to the basic assumption (2.1). We state the theorem on the existence of Busemann functions, both point-to-point and point-to-line. This theorem is proved in Sect. 6. After the theorem we discuss two examples: the exactly solvable case and the percolation cone.

Theorem 3.1 Fix two points $\zeta, \eta \in \mathscr{D}$ such that $\zeta \cdot e_{1} \leq \eta \cdot e_{1}$. Assume that either

(i) $\zeta=\eta=\xi \in \mathscr{E}$ in which case $\zeta=\eta=\xi=\underline{\xi}=\bar{\xi}$, or that

(ii) $[\zeta, \eta]$ is a maximal linear segment of $g_{\mathrm{pp}}$ in which case $[\zeta, \eta]=[\underline{\xi}, \bar{\xi}]$ for all $\xi \in[\zeta, \eta]$.

Then there exists a stationary $L^{1}(\mathbb{P})$ cocycle $\left\{B(x, y): x, y \in \mathbb{Z}^{2}\right\}$ and an event $\Omega_{0}$ with $\mathbb{P}\left(\Omega_{0}\right)=1$ such that the following holds for each $\omega \in \Omega_{0}$. For each sequence $v_{n} \in \mathbb{Z}_{+}^{2}$ such that

$$
\left|v_{n}\right|_{1} \rightarrow \infty \text { and } \zeta \cdot e_{1} \leq \lim _{n \rightarrow \infty} \frac{v_{n} \cdot e_{1}}{\left|v_{n}\right|_{1}} \leq \varlimsup_{n \rightarrow \infty} \frac{v_{n} \cdot e_{1}}{\left|v_{n}\right|_{1}} \leq \eta \cdot e_{1}
$$

we have the limit

$$
B(\omega, x, y)=\lim _{n \rightarrow \infty}\left(G_{x, v_{n}}(\omega)-G_{y, v_{n}}(\omega)\right) \quad \text { for } x, y \in \mathbb{Z}^{2} .
$$

Furthermore, if $h=t\left(e_{1}+e_{2}\right)-\nabla g_{\mathrm{pp}}(\xi)$ for some $t \in \mathbb{R}$ and any (and hence all) $\xi \in[\zeta, \eta]$, we have the limit

$$
B(\omega, 0, z)+h \cdot z=\lim _{n \rightarrow \infty}\left(G_{n}(h)-G_{n-1}(h) \circ T_{z}\right) \quad \text { for } z \in\left\{e_{1}, e_{2}\right\}
$$


The mean of the limit is given by

$$
\nabla g_{\mathrm{pp}}(\xi)=\left(\mathbb{E}\left[B\left(x, x+e_{1}\right)\right], \mathbb{E}\left[B\left(x, x+e_{2}\right)\right]\right) \text { for all } \xi \in[\zeta, \eta]
$$

To paraphrase the theorem, suppose $\xi$ is an exposed point of differentiability of $g_{\mathrm{pp}}$, or $\xi$ lies on a maximal linear segment of $g_{\mathrm{pp}}$ whose endpoints are points of differentiability. Then a Busemann function $B^{\xi}$ exists in direction $\xi$ in the sense that $B^{\xi}(\omega, x, y)$ equals the a.s. limit in (3.2) for any sequence $v_{n} /\left|v_{n}\right|_{1} \rightarrow \xi$ with $\left|v_{n}\right|_{1} \rightarrow \infty$. Furthermore, the $B^{\xi}$ 's match for points $\xi$ on maximal linear segments of $g_{\text {pp }}$ with endpoints in $\mathscr{D}$. The condition on $h$ in the theorem is exactly that $h$ and $\xi$ are dual in the sense of (2.7).

We shall not derive the cocycle properties of $B$ from the limit (3.2). Instead we construct a family of cocycles on an extended space $\widehat{\Omega}=\Omega \times \Omega^{\prime}$ and show that one of these cocycles equals the limit on the right of (3.2).

The Busemann limits (3.2) can also be interpreted as convergence of the last-passage process to a stationary last-passage process, described in Sect. 5.2.

Equation (3.4) was anticipated in [34] (see paragraph after the proof of Theorem 1.13) for Euclidean first passage percolation (FPP) where $g_{\mathrm{pp}}(x, y)=c \sqrt{x^{2}+y^{2}}$. A version of this formula appears also in Theorem 3.5 of [19] for lattice FPP.

The next theorem states that the Busemann functions found in Theorem 3.1 give minimizing cocycles.

Theorem 3.2 Let $\xi \in$ ri $\mathscr{U}$ with $\mathscr{U} \xi=[\underline{\xi}, \bar{\xi}]$ defined in (2.11). Assume that $\underline{\xi}, \xi, \bar{\xi} \in$ $\mathscr{D}$. Let $B^{\xi} \in \mathscr{K}(\Omega)$ be the limit in (3.2) for any sequence $v_{n}$ that satisfies (3.1) for $\zeta=\xi$ and $\eta=\bar{\xi}$. We have $h\left(B^{\xi}\right)=-\nabla g_{\mathrm{pp}}(\xi)$ by (3.4) and (2.12). Define $F(x, y)=h\left(B^{\xi}\right) \cdot(x-y)-B^{\xi}(x, y)$ as in $(2.13)$.

(i) Let $h=h\left(B^{\xi}\right)+(t, t)$ for some $t \in \mathbb{R}$. Then for $\mathbb{P}$-a.e. $\omega$

$$
g_{\mathrm{pl}}(h)=\max _{i \in\{1,2\}}\left\{\omega_{0}+h \cdot e_{i}+F\left(\omega, 0, e_{i}\right)\right\}=t .
$$

In other words, $F$ is a minimizer in (2.14) and the essential supremum vanishes. (ii) For $\mathbb{P}$-a.e. $\omega$

$$
g_{\mathrm{pp}}(\xi)=\max _{i \in\{1,2\}}\left\{\omega_{0}-B^{\xi}\left(\omega, 0, e_{i}\right)-h\left(B^{\xi}\right) \cdot \xi\right\}=-h\left(B^{\xi}\right) \cdot \xi
$$

In other words, $B^{\xi}$ is a minimizer in (2.15) and the essential supremum vanishes.

The condition $h=h\left(B^{\xi}\right)+(t, t)$ for some $t \in \mathbb{R}$ is equivalent to $h$ dual to $\xi$. Every $h$ has a dual $\xi \in$ ri $\mathscr{U}$ as we show in Sect. 4. Consequently, if $g_{\text {pp }}$ is differentiable everywhere on ri $\mathscr{U}$, each $h$ has a minimizing cocycle $F$ that satisfies (3.5) and is obtained by centering a Busemann function. Theorem 3.2 is proved in Sect. 6.

The choice of $i \in\{1,2\}$ in (3.5) and (3.6) depends on $\omega$ and is related to the notion of competition interface. This issue is addressed in the companion paper [26].

Borrowing from homogenization literature (see e.g. page 468 of [3]), a minimizer of (2.14) that removes the essential supremum, that is, a mean zero cocycle that satisfies (3.5), could be called a corrector. 


\subsection{Exactly solvable models}

We illustrate the results in the two exactly solvable cases: the distribution of the mean $m_{0}$ weights $\omega_{x}$ is

exponential: $\mathbb{P}\left\{\omega_{x} \geq t\right\}=e^{-t / m_{0}}$ for $t \geq 0$ with $\sigma^{2}=m_{0}^{2}$,

or geometric: $\mathbb{P}\left\{\omega_{x} \geq k\right\}=\left(1-m_{0}^{-1}\right)^{k}$ for $k \in \mathbb{N}$ with $\sigma^{2}=m_{0}\left(m_{0}-1\right)$.

Calculations behind the claims below are sketched in Sect. 7.1 after the connection with queueing theory has been established.

For both cases in (3.7) the point-to-point limit function is

$$
g_{\mathrm{pp}}(\xi)=m_{0}\left(\xi \cdot e_{1}+\xi \cdot e_{2}\right)+2 \sigma \sqrt{\left(\xi \cdot e_{1}\right)\left(\xi \cdot e_{2}\right)} .
$$

In the exponential case this formula was first derived by Rost [52] (who presented the model in its coupling with TASEP without the last-passage formulation) while early derivations of the geometric case appeared in [16,36,54]. Convex duality (2.7) becomes

$\xi \in \operatorname{ri} \mathscr{U}$ is dual to $h$ if and only if

$$
\exists t \in \mathbb{R}: \quad h=\left(m_{0}+\sigma \sqrt{\xi \cdot e_{1} / \xi \cdot e_{2}}+t, m_{0}+\sigma \sqrt{\xi \cdot e_{2} / \xi \cdot e_{1}}+t\right) .
$$

This in turn gives an explicit formula for $g_{\mathrm{pl}}(h)$.

Since $g_{\text {pp }}$ above is differentiable and strictly concave, all points of ri $\mathscr{U}$ are exposed points of differentiability. Theorem 3.1 implies that Busemann functions (3.2) exist in all directions $\xi \in \mathrm{ri} \mathscr{U}$. They minimize formulas (2.14) and (2.15) as given in (3.5) and (3.6). For each $\xi \in$ ri $\mathscr{U}$ the processes $\left\{B^{\xi}\left(k e_{1},(k+1) e_{1}\right): k \in \mathbb{Z}_{+}\right\}$ and $\left\{B^{\xi}\left(k e_{2},(k+1) e_{2}\right): k \in \mathbb{Z}_{+}\right\}$are i.i.d. processes independent of each other, exponential or geometric depending on the case, with means

$$
\mathbb{E}\left[B^{\xi}\left(k e_{i},(k+1) e_{i}\right)\right]=m_{0}+\sigma \sqrt{\xi \cdot e_{3-i} / \xi \cdot e_{i}}, \quad i \in\{1,2\} .
$$

For the distribution of $B^{\xi}$ see Theorem 8.1 in [14], Section 3.3 in [15], and Sect. 7.1 below.

\subsection{Flat edge in the percolation cone}

In this section we assume that the LPP weights satisfy $\omega_{x} \leq 1$ and $p_{1}=\mathbb{P}\left\{\omega_{0}=1\right\}>$ 0 . The classic Durrett-Liggett flat edge result, sharpened by Marchand, implies that if $p_{1}$ is large enough, $g_{\mathrm{pp}}$ is linear on the percolation cone. By the more recent work of Auffinger-Damron, $g_{\mathrm{pp}}$ is differentiable on the edges. We make a precise statement about this below, after a short detour into oriented percolation.

In oriented site percolation vertices of $\mathbb{Z}^{2}$ are assigned i.i.d. $\{0,1\}$-valued random variables $\left\{\sigma_{z}\right\}_{z \in \mathbb{Z}^{2}}$ with $p_{1}=\mathbb{P}\left\{\sigma_{0}=1\right\}$. For points $u \leq v$ in $\mathbb{Z}^{2}$ we write $u \rightarrow v$ (there 
is an open path from $u$ to $v$ ) if there exists an up-right path $u=x_{0}, x_{1}, \ldots, x_{m}=v$ with $x_{i}-x_{i-1} \in\left\{e_{1}, e_{2}\right\}, m=|v-u|_{1}$, and such that $\sigma_{x_{i}}=1$ for $i=1, \ldots, m$. (The openness of a path does not depend on the weight at the initial point of the path.) The percolation event $\{u \rightarrow \infty\}$ is the existence of an infinite open up-right path from point $u$. There exists a critical threshold $\mathbf{p}_{c} \in(0,1)$ such that if $p_{1}<\mathbf{p}_{c}$ then $\mathbb{P}\{0 \rightarrow \infty\}=0$ and if $p_{1}>\mathbf{p}_{c}$ then $\mathbb{P}\{0 \rightarrow \infty\}>0$. (The facts we need about oriented site percolation are proved in article [20] for oriented edge percolation. The proofs apply to site percolation just as well.)

Let $\mathscr{O}_{n}=\left\{u \in \mathbb{Z}_{+}^{2}:|u|_{1}=n, 0 \rightarrow u\right\}$ denote the set of vertices on level $n$ that can be reached from the origin along open paths. The right edge $a_{n}=\max _{u \in \mathscr{O}_{n}}\left\{u \cdot e_{1}\right\}$ is defined on the event $\left\{\mathscr{O}_{n} \neq \varnothing\right\}$. When $p_{1} \in\left(\mathbf{p}_{c}, 1\right)$ there exists a constant $\beta_{p_{1}} \in$ $(1 / 2,1)$ such that $[20$, eqn. (7) on p. 1005]

$$
\lim _{n \rightarrow \infty} \frac{a_{n}}{n} \mathbb{1}\{0 \rightarrow \infty\}=\beta_{p_{1}} \mathbb{1}\{0 \rightarrow \infty\} \quad \mathbb{P} \text {-a.s. }
$$

Let $\bar{\eta}=\left(\beta_{p_{1}}, 1-\beta_{p_{1}}\right)$ and $\underline{\eta}=\left(1-\beta_{p_{1}}, \beta_{p_{1}}\right)$. The percolation cone is the set $\left\{\xi \in \mathbb{R}_{+}^{2}: \xi /|\xi|_{1} \in[\eta, \bar{\eta}]\right\}$. The next theorem is proved by associating an oriented site percolation process to the LPP process by defining $\sigma_{x}=\mathbb{1}\left\{\omega_{x}=1\right\}$.

Theorem 3.3 Assume that $\left\{\omega_{x}\right\}_{x \in \mathbb{Z}^{2}}$ are i.i.d., $\mathbb{E}\left|\omega_{0}\right|^{p}<\infty$ for some $p>2$ and $\omega_{x} \leq 1$. Suppose $\mathbf{p}_{c}<p_{1}=\mathbb{P}\left\{\omega_{0}=1\right\}<1$. Let $\xi \in \mathscr{U}$. Then $g_{\mathrm{pp}}(\xi) \leq 1$, and $g_{\mathrm{pp}}(\xi)=1$ if and only if $\xi \in[\eta, \bar{\eta}]$. The endpoints $\eta$ and $\bar{\eta}$ are points of differentiability of $g_{\mathrm{pp}}$.

Theorem 3.3 above summarizes a development carried out for undirected firstpassage percolation in articles [4,21,42]. A proof of Theorem 3.3 for the corner growth model, adapted from the earlier arguments, is in Appendix D of [25]. As a corollary, our results that assume differentiable endpoints of a maximal linear segment are valid for the percolation cone.

Theorem 3.4 Assume (2.1), $\omega_{x} \leq 1$ and $\mathbf{p}_{c}<p_{1}=\mathbb{P}\left\{\omega_{0}=1\right\}<1$. There exists a stationary $L^{1}(\mathbb{P})$ cocycle $\left\{B(x, y): x, y \in \mathbb{Z}^{2}\right\}$ and an event $\Omega_{0}$ with $\mathbb{P}\left(\Omega_{0}\right)=1$ such that the following statements hold for each $\omega \in \Omega_{0}$. Let $v_{n} \in \mathbb{Z}_{+}^{2}$ be a sequence such that

$$
\left|v_{n}\right|_{1} \rightarrow \infty \text { and } 1-\beta_{p_{1}} \leq \lim _{n \rightarrow \infty} \frac{v_{n} \cdot e_{1}}{\left|v_{n}\right|_{1}} \leq \varlimsup_{n \rightarrow \infty} \frac{v_{n} \cdot e_{1}}{\left|v_{n}\right|_{1}} \leq \beta_{p_{1}}
$$

Then

$$
B(\omega, x, y)=\lim _{n \rightarrow \infty}\left(G_{x, v_{n}}(\omega)-G_{y, v_{n}}(\omega)\right)
$$

for all $x, y \in \mathbb{Z}^{2}$. Furthermore, $\mathbb{E}\left[B\left(x, x+e_{1}\right)\right]=\mathbb{E}\left[B\left(x, x+e_{2}\right)\right]=1$.

Continuing with the assumptions of Theorem 3.4, we develop a more explicit formula for the Busemann function. Let

$$
\psi(\omega)=\inf _{x_{0, \infty}: x_{0}=0} \sum_{k=0}^{\infty}\left(1-\omega_{x_{k}}\right)
$$


where the infimum is over all infinite up-right paths that start at $x_{0}=0 . \psi$ is measurable because it is the nondecreasing limit of $n-G_{n}(0)$ as $n \rightarrow \infty$. As part of verifying this we take a convergent subsequence of maximizing paths for $G_{n}(0)$ and establish the existence of a path $\bar{x}_{0, \infty}$ such that

$$
\psi(\omega)=\sum_{k=0}^{\infty}\left(1-\omega_{\bar{x}_{k}}\right)=\lim _{n \rightarrow \infty}\left(n-G_{0, \bar{x}_{n}}\right) .
$$

$\bar{x}_{0, \infty}$ must be a geodesic, that is, any segment $\bar{x}_{m, n}$ for $0 \leq m<n<\infty$ must give the maximal passage time $G_{\bar{x}_{m}, \bar{x}_{n}}$ because otherwise there is a better path.

By [20, eqn. (3) on p. 1028] applied to oriented site percolation, under $p_{1}>\mathbf{p}_{c}$ the event $\cup_{0 \leq k \leq n}\{(k, n-k) \rightarrow \infty\}$ fails with probability at most $e^{-\gamma n}$ for a constant $\gamma>0$. On this event $\psi \leq(1-c) n$. (Recall that $\omega_{x} \geq c$ a.s. is part of assumption (2.1).) Consequently $\psi$ has an exponential moment and in particular is almost surely finite.

Theorem 3.5 Under the assumptions of Theorem 3.4, the Busemann function B of the percolation cone in (3.11) is given by

$$
B(\omega, x, y)=(y-x) \cdot\left(e_{1}+e_{2}\right)+\psi\left(T_{y} \omega\right)-\psi\left(T_{x} \omega\right)
$$

Proof Let $x_{0, \infty}^{\prime}$ be a path that achieves the infimum over paths that start at $x$, in the environment $\omega$ :

$$
\psi(x, \omega)=\psi\left(T_{x} \omega\right)=\inf _{x_{0, \infty}: x_{0}=x} \sum_{k=0}^{\infty}\left(1-\omega_{x_{k}}\right)=\sum_{k=0}^{\infty}\left(1-\omega_{x_{k}^{\prime}}\right) .
$$

We argue that sequence $x_{n}^{\prime}$ must satisfy (3.10). To get a contradiction, suppose that $x_{n_{i}}^{\prime} \cdot e_{1}>\left|x_{n_{i}}^{\prime}\right|_{1}\left(\beta_{p_{1}}+\varepsilon\right)$ for some $\varepsilon>0$ and a subsequence $n_{i}$. Then by the shape theorem (2.5) and the part of Theorem 3.3 that says $g_{\mathrm{pp}}<1$ away from the percolation cone, $\overline{\lim } n_{i}^{-1} G_{x, x_{n_{i}}^{\prime}} \leq 1-\delta$ for some $\delta>0$. We have a contradiction:

$$
\psi(x, \omega) \geq \sum_{k=0}^{n_{i}-1}\left(1-\omega_{x_{k}^{\prime}}\right)=n_{i}-G_{x, x_{n_{i}}^{\prime}} \nearrow \infty \quad \text { as } i \rightarrow \infty .
$$

Now we can take the limit in (3.11) along $x_{n}^{\prime}$. Let $m_{n}^{\prime}=\left|x_{n}^{\prime}-x\right|_{1}$ and $m_{n}^{\prime \prime}=$ $\left|x_{n}^{\prime}-y\right|_{1}$. Take $n$ large enough so that $x_{n}^{\prime} \geq x \vee y$ in coordinatewise order. Use $G_{y, x_{n}^{\prime}} \leq G_{m_{n}^{\prime \prime}}(0) \circ T_{y}$ to write

$$
\begin{aligned}
G_{x, x_{n}^{\prime}}-G_{y, x_{n}^{\prime}} \geq & \left(x_{n}^{\prime}-x\right) \cdot\left(e_{1}+e_{2}\right)-\left[m_{n}^{\prime}-G_{x, x_{n}^{\prime}}\right] \\
& -\left(x_{n}^{\prime}-y\right) \cdot\left(e_{1}+e_{2}\right)+\left[m_{n}^{\prime \prime}-G_{m_{n}^{\prime \prime}}(0) \circ T_{y}\right]
\end{aligned}
$$


and take a limit to get

$$
B(\omega, x, y)=\lim _{n \rightarrow \infty}\left(G_{x, x_{n}^{\prime}}-G_{y, x_{n}^{\prime}}\right) \geq(y-x) \cdot\left(e_{1}+e_{2}\right)-\psi\left(T_{x} \omega\right)+\psi\left(T_{y} \omega\right) .
$$

Switch around $x$ and $y$ and use $B(\omega, x, y)=-B(\omega, y, x)$ to get the opposite inequality. The proof is complete.

Remark 3.6 (Weak disorder in the percolation cone) At inverse temperature $\beta \in$ $(0, \infty)$, the point-to-line partition function of the directed polymer model is

$$
Z_{n}=\sum_{x_{0, n-1}} 2^{-n+1} e^{\beta \sum_{k=0}^{n-1} \omega_{x_{k}}}
$$

where the sum is over admissible paths $x_{0, n-1}$ that start at $x_{0}=0$. The normalized partition function $W_{n}=Z_{n} / \mathbb{E} Z_{n}$ is a positive martingale and has an a.s. limit $W_{n} \rightarrow$ $W_{\infty}$. By definition, the model is in weak disorder if $\mathbb{P}\left(W_{\infty}>0\right)=1$ and otherwise in strong disorder [33, p. 208]. It is known that the $1+1$ dimensional directed polymer is in strong disorder at all positive $\beta$-values, as long as $\omega_{0}$ has finite exponential moments. (See [17, Thm. 2.3(b)] and [12, Thm. 1.1]).

The zero-temperature limit of the polymer is the point-to-line last-passage model: $e^{G_{n}(0)}=\lim _{\beta \rightarrow \infty} Z_{n}^{1 / \beta}$, while $\lim _{\beta \rightarrow \infty} W_{n}^{1 / \beta}=e^{G_{n}(0)-n}$. Process $e^{G_{n}(0)-n}$ is no longer a martingale but it is a supermartingale (because $G_{n}(0) \leq G_{n-1}(0)+1$ ) and, as we have seen, converges a.s. to $e^{-\psi}$. Above we observed that $\psi<\infty$ under $p_{1}>\mathbf{p}_{c}$. So the martingale criterion suggests that the model is in weak disorder.

We can observe two other markers of weak disorder. The fluctuation exponent of $G_{n}(0)$ is zero, since without any normalization, $G_{n}(0)-n g_{\mathrm{pl}}(0)=G_{n}(0)-n$ converges to a finite random quantity. Furthermore, the centered cocycle $F(\omega, x, y)=$ $h(B) \cdot(x-y)-B(\omega, x, y)$ that minimizes in Theorem 3.2(i) is a gradient: $F(\omega, x, y)=$ $\psi\left(T_{x} \omega\right)-\psi\left(T_{y} \omega\right)$. It is in general true in weak disorder that the minimizer of the variational formula for the quenched point-to-line free energy is a gradient [51, Thm. 2.8], though it is currently an open question whether this is a characterization of weak disorder.

Notice for the remainder of the paper No assumptions on the weights beyond (2.1) and no regularity assumptions on the shape function $g_{\mathrm{pp}}$ are used, except when otherwise stated.

\section{Duality}

By homogeneity we can represent $g_{\text {pp }}$ by a single variable function. A way of doing this that ties in naturally with the queuing theory arguments we use later is to define

$$
\gamma(s)=g_{\mathrm{pp}}(1, s)=g_{\mathrm{pp}}(s, 1) \text { for } 0 \leq s<\infty
$$


Function $\gamma$ is real-valued, continuous and concave. Consequently one-sided derivatives $\gamma^{\prime}(s \pm)$ exist and are monotone: $\gamma^{\prime}\left(s_{0}+\right) \geq \gamma^{\prime}\left(s_{1}-\right) \geq \gamma^{\prime}\left(s_{1}+\right)$ for $0 \leq s_{0}<s_{1}$. Symmetry and homogeneity of $g_{\text {pp }}$ give $\gamma(s)=s \gamma\left(s^{-1}\right)$.

Lemma 4.1 The derivatives satisfy $\gamma^{\prime}(s \pm)>m_{0}$ for all $s \in \mathbb{R}_{+}, \gamma^{\prime}(0+)=\infty$, and $\gamma^{\prime}(\infty-) \equiv \lim _{s \rightarrow \infty} \gamma^{\prime}(s \pm)=\gamma(0)=m_{0}$.

Proof The shape universality at the boundary of $\mathbb{R}_{+}^{2}$ by Martin [43, Theorem 2.4] says that

$$
\gamma(s)=m_{0}+2 \sigma \sqrt{s}+o(\sqrt{s}) \text { as } s \searrow 0 \text {. }
$$

This gives $\gamma(0)=m_{0}$ and $\gamma^{\prime}(0+)=\infty$. Lastly,

$$
\gamma^{\prime}(\infty-)=\lim _{s \rightarrow \infty} s^{-1} \gamma(s)=\lim _{s \rightarrow \infty} \gamma\left(s^{-1}\right)=\gamma(0)=m_{0} .
$$

Martin's asymptotic (4.2) and $\gamma(s)=s \gamma\left(s^{-1}\right)$ give

$$
\gamma(s)=s m_{0}+2 \sigma \sqrt{s}+o(\sqrt{s}) \text { as } s \nearrow \infty .
$$

This is incompatible with having $\gamma^{\prime}(s)=m_{0}$ for $s \geq s_{0}$ for any $s_{0}<\infty$.

The lemma above has two important geometric consequences:

every linear segment of $g_{\text {pp }}$ must lie in the interior ri $\mathscr{U}$, and the boundary $\left\{\xi \in \mathbb{R}_{+}^{2}: g_{\mathrm{pp}}(\xi)=1\right\}$ of the limit shape is asymptotic to the axes.

Define

$$
f(\alpha)=\sup _{s \geq 0}\{\gamma(s)-s \alpha\} \text { for } m_{0}<\alpha<\infty .
$$

Remark 4.2 (Queueing interpretations) The quantities introduced in this section have natural interpretations in a queueing context. The queueing connection of LPP in terms of tandem service stations goes as follows. Imagine a queueing system with customers labeled $0,1, \ldots, m$ and service stations labeled $0,1, \ldots, n$. The random weight $\omega_{i, j}$ is the service time of customer $i$ at station $j$. At time $t=0$ all customers are lined up at service station 0 . Customers proceed through the system in order, obeying FIFO (first-in-first-out) discipline, and joining the queue at station $j+1$ as soon as service at station $j$ is complete. Then for each $0 \leq k \leq m$ and $0 \leq \ell \leq n, G_{(0,0),(k, \ell)}$ is the time when customer $k$ enters service at station $\ell$ and $G_{(0,0),(k, \ell)}+\omega_{k, \ell}$ is the time when customer $k$ departs station $\ell$ and joins the end of the queue at station $\ell+1$. Among the seminal references for these ideas are $[29,44]$.

In Sect. 7 we make use of a queueing system that operates in this manner but is stationary in space and time, and the sequences of customers and stations are biinfinite. In this setting $\alpha \in\left(m_{0}, \infty\right)$ is the mean interarrival (and interdeparture) time 
of customers at each queue, and it parametrizes the stationary distributions of the system. $f(\alpha)$ is the mean sojourn time, that is, the time between arrival and departure of a particular customer at a particular station.

Lemma 4.3 Function $f$ is a strictly decreasing, continuous and convex involution of the interval $\left(m_{0}, \infty\right)$ onto itself, with limits $f\left(m_{0}+\right)=\infty$ and $f(\infty-)=m_{0}$. That $f$ is an involution means that $f(f(\alpha))=\alpha$.

Proof Asymptotics (4.2) and (4.3) imply that $m_{0}<f(\alpha)<\infty$ for all $\alpha>m_{0}$ and also that the supremum in (4.5) is attained at some $s$. Furthermore, $\alpha<\beta$ implies $f(\beta)=\gamma\left(s_{0}\right)-s_{0} \beta$ with $s_{0}>0$ and $f(\beta)<\gamma\left(s_{0}\right)-s_{0} \alpha \leq f(\alpha)$. As a supremum of linear functions $f$ is convex, and hence continuous on the open interval $\left(m_{0}, \infty\right)$.

We show how the symmetry of $g_{\mathrm{pp}}$ implies that $f$ is an involution. By concavity of $\gamma$

$$
f(\alpha)=\gamma(s)-s \alpha \quad \text { if and only if } \alpha \in\left[\gamma^{\prime}(s+), \gamma^{\prime}(s-)\right]
$$

and by Lemma 4.1 the intervals on the right cover $\left(m_{0}, \infty\right)$. Since $f$ is strictly decreasing the above is the same as

$$
\alpha=\gamma\left(s^{-1}\right)-s^{-1} f(\alpha) \text { if and only if } f(\alpha) \in\left[f\left(\gamma^{\prime}(s-)\right), f\left(\gamma^{\prime}(s+)\right)\right] .
$$

Differentiating $\gamma(s)=s \gamma\left(s^{-1}\right)$ gives

$$
\gamma^{\prime}(s \pm)=\gamma\left(s^{-1}\right)-s^{-1} \gamma^{\prime}\left(s^{-1} \mp\right) .
$$

By (4.6) and (4.8) the condition in (4.7) can be rewritten as

$$
f(\alpha) \in\left[\gamma(s)-s \gamma^{\prime}(s-), \gamma(s)-s \gamma^{\prime}(s+)\right]=\left[\gamma^{\prime}\left(s^{-1}+\right), \gamma^{\prime}\left(s^{-1}-\right)\right] .
$$

Combining this with (4.6) and (4.7) shows that $\alpha=f(f(\alpha))$. The claim about the limits follows from $f$ being a decreasing involution.

Extend these functions to the entire real line by $\gamma(s)=-\infty$ when $s<0$ and $f(\alpha)=\infty$ when $\alpha \leq m_{0}$. Then convex duality gives

$$
\gamma(s)=\inf _{\alpha>m_{0}}\{f(\alpha)+s \alpha\}
$$

The natural bijection between $s \in(0, \infty)$ and $\xi \in$ ri $\mathscr{U}$ that goes together with (4.1) is

$$
s=\xi \cdot e_{1} / \xi \cdot e_{2} .
$$

Then direct differentiation, (4.6) and (4.8) give

$$
\nabla g_{\mathrm{pp}}(\xi \pm)=\left(\gamma^{\prime}(s \pm), \gamma^{\prime}\left(s^{-1} \mp\right)\right)=\left(\gamma^{\prime}(s \pm), f\left(\gamma^{\prime}(s \pm)\right)\right)
$$


Since $f$ is linear on $\left[\gamma^{\prime}(s+), \gamma^{\prime}(s-)\right]$, we get the following connection between the gradients of $g_{\mathrm{pp}}$ and the graph of $f:$ for $\xi \in$ ri $\mathscr{U}$,

$$
\left[\nabla g_{\mathrm{pp}}(\xi+), \nabla g_{\mathrm{pp}}(\xi-)\right]=\left\{(\alpha, f(\alpha)): \alpha \in\left[\gamma^{\prime}(s+), \gamma^{\prime}(s-)\right]\right\}
$$

The next theorem details the duality between tilts $h$ and velocities $\xi$. It is needed only for Sect. 5.3 where we solve the variational formulas.

Theorem 4.4 (i) Let $h \in \mathbb{R}^{2}$. There exists a unique $t=t(h) \in \mathbb{R}$ such that

$$
h-t\left(e_{1}+e_{2}\right) \in-\left[\nabla g_{\mathrm{pp}}(\xi+), \nabla g_{\mathrm{pp}}(\xi-)\right]
$$

for some $\xi \in$ ri $\mathscr{U}$. The set of $\xi$ for which (4.14) holds is a nonempty (but possibly degenerate) line segment $[\underline{\xi}(h), \bar{\xi}(h)] \subset$ ri $\mathscr{U}$. If $\underline{\xi}(h) \neq \bar{\xi}(h)$ then $[\underline{\xi}(h), \bar{\xi}(h)]$ is a maximal linear segment of $g_{\mathrm{pp}}$.

(ii) $\xi \in$ ri $\mathscr{U}$ and $h \in \mathbb{R}^{2}$ satisfy duality (2.7) if and only if (4.14) holds.

Proof The graph $\left\{(\alpha, f(\alpha)): \alpha>m_{0}\right\}$ is strictly decreasing with limits $f\left(m_{0}+\right)=$ $\infty$ and $f(\infty-)=m_{0}$. Since every 45 degree diagonal intersects it at anique point, the equation

$$
h=-(\alpha, f(\alpha))+t\left(e_{1}+e_{2}\right)
$$

defines a bijection $\mathbb{R}^{2} \ni h \longleftrightarrow(\alpha, t) \in\left(m_{0}, \infty\right) \times \mathbb{R}$ illustrated in Fig. 2. Combining this with (4.13) shows that (4.14) happens for a unique $t$ and for at least one $\xi \in$ ri $\mathscr{U}$.

Once $h$ and $t=t(h)$ are given, the geometry of the gradients ((4.12), (4.13) and limits (2.9)) can be used to argue the claims about the $\xi$ that satisfy (4.14). This proves part (i).

Fig. 2 The graph of $f$ and bijection (4.15) between $(\alpha, t)$ and $h$

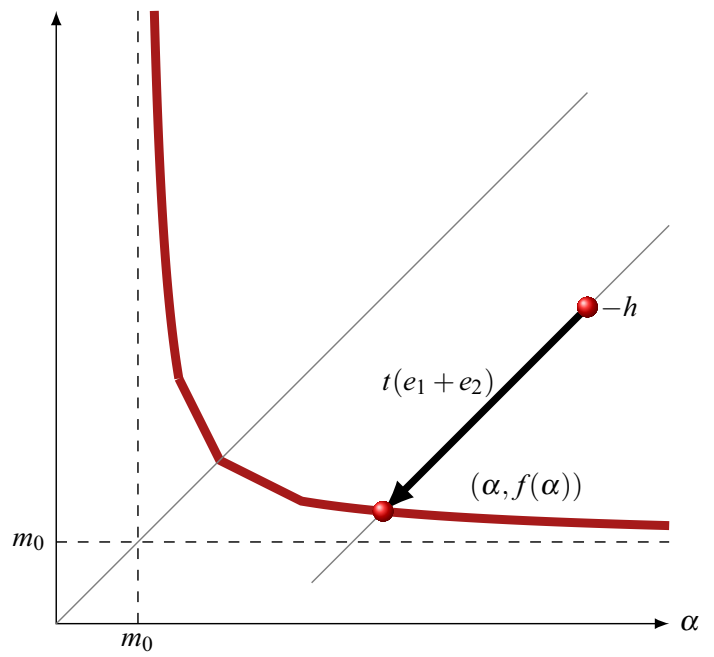


That $h$ of the form (4.14) is dual to $\xi$ follows readily from the fact that gradients are dual and $g_{\mathrm{pl}}\left(h+t\left(e_{1}+e_{2}\right)\right)=g_{\mathrm{pl}}(h)+t$ (this last from Definition (2.4)).

Note the following general facts for any $q \in\left[\nabla g_{\mathrm{pp}}(\zeta+), \nabla g_{\mathrm{pp}}(\zeta-)\right]$. By concavity $g_{\mathrm{pp}}(\eta) \leq g_{\mathrm{pp}}(\zeta)+q \cdot(\eta-\zeta)$ for all $\eta$. Combining this with homogeneity gives $g_{\mathrm{pp}}(\zeta)=q \cdot \zeta$. Together with duality (2.6) we have

$$
g_{\mathrm{pl}}(-q)=0 \text { for } q \in \bigcup_{\zeta \in \mathrm{ri} \mathscr{U}}\left[\nabla g_{\mathrm{pp}}(\zeta+), \nabla g_{\mathrm{pp}}(\zeta-)\right]
$$

It remains to show that if $h$ is dual to $\xi$ then it satisfies (4.14). Let $(\alpha, t)$ be determined by (4.15). From the last two paragraphs

$$
g_{\mathrm{pl}}(h)=g_{\mathrm{pl}}(-\alpha,-f(\alpha))+t=t .
$$

Let $s=\xi \cdot e_{1} / \xi \cdot e_{2}$ so that

$$
g_{\mathrm{pp}}(\xi)+h \cdot \xi=\frac{\gamma(s)}{1+s}-\frac{\alpha s+f(\alpha)}{1+s}+t .
$$

Thus duality $g_{\mathrm{pl}}(h)=g_{\mathrm{pp}}(\xi)+h \cdot \xi$ implies $\gamma(s)=\alpha s+f(\alpha)$ which happens if and only if $\alpha \in\left[\gamma^{\prime}(s+), \gamma^{\prime}(s-)\right]$. (4.13) now implies (4.14).

\section{Stationary cocycles}

In this section we describe the stationary cocycles, then show how these define stationary last-passage percolation processes and also solve the variational formulas for $g_{\mathrm{pp}}(\xi)$ and $g_{\mathrm{pl}}(h)$. Assumption (2.1) is in force but no other assumptions are made.

\subsection{Existence and properties of stationary cocycles}

By appeal to queueing fixed points, in Sect. 7 we construct a family of cocycles $\left\{B_{ \pm}^{\xi}\right\}_{\xi \in \text { ri } \mathscr{U}}$ on an extended space $\widehat{\Omega}=\Omega \times \Omega^{\prime}=\Omega \times \mathbb{R}^{\{1,2\} \times \mathscr{A}_{0} \times \mathbb{Z}^{2}}$ where $\mathscr{A}_{0}$ is a countable subset of the interval $\left(m_{0}, \infty\right)$, defined in (7.15) below. Generic elements of $\widehat{\Omega}$ are denoted by $\widehat{\omega}=\left(\omega, \omega^{\prime}\right)$ where $\omega=\left(\omega_{x}\right)_{x \in \mathbb{Z}^{2}} \in \Omega=\mathbb{R}^{\mathbb{Z}^{2}}$ is the original weight

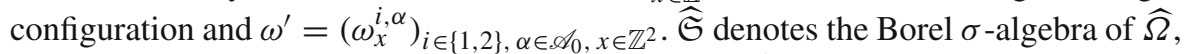
and in this context $\mathfrak{S}$ denotes the sub- $\sigma$-algebra of $\widehat{\mathfrak{S}}$ generated by the projection $\widehat{\omega} \mapsto \omega$. Spatial translations act in the usual manner: $\left(T_{x} \widehat{\omega}\right)_{y}=\widehat{\omega}_{x+y}$ for $x, y \in \mathbb{Z}^{2}$ where $\widehat{\omega}_{x}=\left(\omega_{x}, \omega_{x}^{\prime}\right)=\left(\omega_{x},\left(\omega_{x}^{i, \alpha}\right)_{i \in\{1,2\}, \alpha \in \mathscr{A}_{0}}\right)$.

Definition 2.1 of a cocycle makes no reference to the LPP process. The key feature that connects cocycles with the last-passage weights is captured in the next definition. It was isolated in our previous paper [27] that developed the cocycle set-up for general polymer and percolation models. This property is behind all our applications of the cocycles: construction of stationary LPP in Sect. 5.2 which in turn is used to prove 
Busemann limits, identification of minimizers of variational formulas in Sect. 5.3, and study of geodesics in [26].

Definition 5.1 A stationary $L^{1}$ cocycle $B$ on $\widehat{\Omega}$ recovers potential $V$ if

$$
V(\widehat{\omega})=\min _{i \in\{1,2\}} B\left(\widehat{\omega}, 0, e_{i}\right) \text { for } \widehat{\mathbb{P}} \text {-a.e. } \widehat{\omega}
$$

In our present case the potential $V: \widehat{\Omega} \rightarrow \mathbb{R}$ is simply $V(\widehat{\omega})=\omega_{0}$, the last-passage weight at the origin.

The next theorem gives the existence statement and summarizes the properties of these cocycles. This is the only place where our proofs use the assumption $\mathbb{P}\left(\omega_{0} \geq\right.$ $c)=1$, and the only reason is that the queueing results we reference have been proved only for $\omega_{0} \geq 0$. In part (i) below we use this notation: for a finite or infinite set $I \subset \mathbb{Z}^{2}, I^{<}=\left\{x \in \mathbb{Z}^{2}: x \nsucceq z \forall z \in I\right\}$ is the set of lattice points that do not lie on a ray from $I$ at an angle in $[0, \pi / 2]$. For example, if $I=\{0, \ldots, m\} \times\{0, \ldots, n\}$ then $I^{<}=\mathbb{Z}^{2} \backslash \mathbb{Z}_{+}^{2}$.

Theorem 5.2 There exist real-valued Borel functions $B_{+}^{\xi}(\widehat{\omega}, x, y)$ and $B_{-}^{\xi}(\widehat{\omega}, x, y)$ of $(\widehat{\omega}, \xi, x, y) \in \widehat{\Omega} \times$ ri $\mathscr{U} \times \mathbb{Z}^{2} \times \mathbb{Z}^{2}$ and a translation invariant Borel probability measure $\widehat{\mathbb{P}}$ on $(\widehat{\Omega}, \widehat{\mathfrak{S}})$ such that the following properties hold.

(i) Under $\widehat{\mathbb{P}}$, the marginal distribution of the configuration $\omega$ is the i.i.d. measure $\mathbb{P}$ specified in assumption (2.1). For each $\xi \in \mathrm{ri} \mathscr{U}$ and \pm , the $\mathbb{R}^{3}$-valued process $\left\{\psi_{x}^{ \pm, \xi}\right\}_{x \in \mathbb{Z}^{2}}$ defined by

$$
\psi_{x}^{ \pm, \xi}(\widehat{\omega})=\left(\omega_{x}, B_{ \pm}^{\xi}\left(\widehat{\omega}, x, x+e_{1}\right), B_{ \pm}^{\xi}\left(\widehat{\omega}, x, x+e_{2}\right)\right)
$$

is separately ergodic under both translations $T_{e_{1}}$ and $T_{e_{2}}$. For any $I \subset \mathbb{Z}^{2}$, the variables

$$
\left\{\left(\omega_{x}, B_{+}^{\xi}\left(\widehat{\omega}, x, x+e_{i}\right), B_{-}^{\xi}\left(\widehat{\omega}, x, x+e_{i}\right)\right): x \in I, \xi \in \operatorname{ri} \mathscr{U}, i \in\{1,2\}\right\}
$$

are independent of $\left\{\omega_{x}: x \in I^{<}\right\}$.

(ii) Each process $B_{ \pm}^{\xi}=\left\{B_{ \pm}^{\xi}(x, y)\right\}_{x, y \in \mathbb{Z}^{2}}$ is a stationary $L^{1}(\widehat{\mathbb{P}})$ cocycle (Definition 2.1) that recovers the potential (Definition 5.1):

$$
\omega_{x}=B_{ \pm}^{\xi}\left(\widehat{\omega}, x, x+e_{1}\right) \wedge B_{ \pm}^{\xi}\left(\widehat{\omega}, x, x+e_{2}\right) \quad \widehat{\mathbb{P}}-\text { a.s. }
$$

The mean vectors $h_{ \pm}(\xi)=h\left(B_{ \pm}^{\xi}\right)$ defined by (2.12) satisfy

$$
-h_{ \pm}(\xi)=\left(\widehat{\mathbb{E}}\left[B_{ \pm}^{\xi}\left(x, x+e_{1}\right)\right], \widehat{\mathbb{E}}\left[B_{ \pm}^{\xi}\left(x, x+e_{2}\right)\right]\right)=\nabla g_{\mathrm{pp}}(\xi \pm)
$$

and are dual to velocity $\xi$ as in (2.7). 
(iii) No two distinct cocycles have a common tilt vector. That is, if $h_{+}(\xi)=h_{-}(\zeta)$ then

$$
B_{+}^{\xi}(\widehat{\omega}, x, y)=B_{-}^{\zeta}(\widehat{\omega}, x, y) \quad \forall \widehat{\omega} \in \widehat{\Omega}, x, y \in \mathbb{Z}^{2}
$$

and similarly for all four combinations of \pm and $\xi, \zeta$. These equalities hold for all $\widehat{\omega}$ without an almost sure modifier because they come directly from the construction. In particular, if $\xi \in \mathscr{D}$ then

$$
B_{+}^{\xi}(\widehat{\omega}, x, y)=B_{-}^{\xi}(\widehat{\omega}, x, y)=B^{\xi}(\widehat{\omega}, x, y) \quad \forall \widehat{\omega} \in \widehat{\Omega}, x, y \in \mathbb{Z}^{2},
$$

where the second equality defines the cocycle $B^{\xi}$.

(iv) There exists an event $\widehat{\Omega}_{0}$ with $\widehat{\mathbb{P}}\left(\widehat{\Omega}_{0}\right)=1$ and such that (a) and (b) below hold for all $\widehat{\omega} \in \widehat{\Omega}_{0}, x, y \in \mathbb{Z}^{2}$ and $\xi, \zeta \in$ ri $\mathscr{U}$.

(a) Monotonicity: if $\xi \cdot e_{1}<\zeta \cdot e_{1}$ then

$$
\begin{aligned}
& B_{-}^{\xi}\left(\widehat{\omega}, x, x+e_{1}\right) \geq B_{+}^{\xi}\left(\widehat{\omega}, x, x+e_{1}\right) \geq B_{-}^{\zeta}\left(\widehat{\omega}, x, x+e_{1}\right) \\
& \text { and } \quad B_{-}^{\xi}\left(\widehat{\omega}, x, x+e_{2}\right) \leq B_{+}^{\xi}\left(\widehat{\omega}, x, x+e_{2}\right) \leq B_{-}^{\zeta}\left(\widehat{\omega}, x, x+e_{2}\right)
\end{aligned}
$$

(b) Right continuity: if $\xi_{n} \cdot e_{1} \searrow \zeta \cdot e_{1}$ then

$$
\lim _{n \rightarrow \infty} B_{ \pm}^{\xi_{n}}(\widehat{\omega}, x, y)=B_{+}^{\zeta}(\widehat{\omega}, x, y)
$$

(v) Left continuity at a fixed $\zeta \in \mathrm{ri} \mathscr{U}$ : there exists an event $\widehat{\Omega}^{(\zeta)}$ with $\widehat{\mathbb{P}}\left(\widehat{\Omega}^{(\zeta)}\right)=1$ and such that for any sequence $\xi_{n} \cdot e_{1} \nearrow \zeta \cdot e_{1}$

$$
\lim _{n \rightarrow \infty} B_{ \pm}^{\xi_{n}}(\widehat{\omega}, x, y)=B_{-}^{\zeta}(\widehat{\omega}, x, y) \quad \text { for } \widehat{\omega} \in \widehat{\Omega}^{(\zeta)}, x, y \in \mathbb{Z}^{d}
$$

Limits (5.5) and (5.6) hold also in $L^{1}(\widehat{\mathbb{P}})$ due to inequalities (5.4).

The conditional expectations $\widehat{\mathbb{E}}\left[B_{ \pm}^{\xi}(x, y) \mid \mathfrak{S}\right]$ are cocycles that are functions of $\omega$ alone, but the conditioning may destroy crucial property (5.2) that relates the cocycles to the percolation. Some mild additional regularity on $g_{\text {pp }}$ guarantees that all cocycles $\widehat{\omega} \mapsto B_{ \pm}^{\xi}(\widehat{\omega}, x, y)$ are in fact $\mathfrak{S}$-measurable. This theorem is proved in Sect. 6 .

Theorem 5.3 Assume that $g_{\mathrm{pp}}$ is differentiable at the endpoints of its linear segments (if any). Then all cocycles $\left\{B_{ \pm}^{\xi}(x, y)\right\}_{\xi \in \mathrm{ri} \mathscr{U}, x, y \in \mathbb{Z}^{2}}$ from Theorem 5.2 are measurable with respect to (the completion of) $\mathfrak{S}$.

Remark 5.4 The cocycle construction of Theorem 5.2 undertaken in Sect. 7 utilizes a countable dense subset $\mathscr{U}_{0}$ of $\mathscr{U}$ such that, for $\xi \in \mathscr{U}_{0}$, nearest-neighbor cocycle values are coordinate projections $B_{ \pm}^{\xi}\left(\widehat{\omega}, x, x+e_{i}\right)=\omega_{x}^{i, \gamma^{\prime}(s \pm)}$ where $s$ is defined by (4.11). $\mathscr{U}_{0}$ contains all points of nondifferentiability and endpoints of linear segments of $g_{\text {pp }}$. For $\zeta \in($ ri $\mathscr{U}) \backslash \mathscr{U}_{0}$ we define $B^{\zeta}=B_{ \pm}^{\zeta}$ through right limits from $\left\{B_{ \pm}^{\xi}\right\}_{\xi \in \mathscr{U}_{0}}$. 
This is behind Theorem 5.2(iii)-(iv). Monotonicity (5.4) simultaneously for all $B_{ \pm}^{\xi}$ outside a single null set will be convenient for constructing geodesics in [26].

The cocycle properties and (5.2) can also be arranged to hold simultaneously for all $B_{ \pm}^{\xi}$ outside a single null set, if so desired. But for left and right limits to agree at a particular $\zeta$ we have to allow for the exceptional $\widehat{\mathbb{P}}$-null event $\left(\widehat{\Omega}^{(\zeta)}\right)^{c}$ that is specific to $\zeta$. Thus left limit (5.6) is not claimed for all $\zeta$ outside a single null set.

Other conventions are possible in the construction. We could extend $B_{ \pm}^{\xi}$ from $\mathscr{U}_{0}$ so that $B_{+}^{\xi}$ is right-continuous and $B_{-}^{\xi}$ left-continuous in $\xi$. Monotonicity (5.4) would still hold outside a single null set, but $B_{+}^{\xi}=B_{-}^{\xi}$ would be only almost surely true for a given $\xi \in \mathscr{E}$, instead of identically true.

\subsection{Stationary last-passage percolation}

Fix a cocycle $B(\widehat{\omega}, x, y)=B_{+}^{\xi}(\widehat{\omega}, x, y)$ or $B_{-}^{\xi}(\widehat{\omega}, x, y)$ from Theorem 5.2. Fix a point $v \in \mathbb{Z}^{2}$ that will serve as an origin. By part (i) of Theorem 5.2, the weights $\left\{\omega_{x}: x \leq\right.$ $\left.v-e_{1}-e_{2}\right\}$ are independent of $\left\{B\left(v-(k+1) e_{i}, v-k e_{i}\right): k \in \mathbb{Z}_{+}, i \in\{1,2\}\right\}$. These define a stationary last-passage percolation process in the third quadrant relative to the origin $v$, in the following sense. Define passage times $G_{u, v}^{\mathrm{NE}}$ that use the cocycle as edge weights on the north and east boundaries and weights $\omega_{x}$ in the bulk $x \leq v-e_{1}-e_{2}$ :

$$
\begin{aligned}
G_{u, v}^{\mathrm{NE}} & =B(u, v) \text { for } u \in\left\{v-k e_{i}: k \in \mathbb{Z}_{+}, i \in\{1,2\}\right\} \\
\text { and } \quad G_{u, v}^{\mathrm{NE}} & =\omega_{u}+G_{u+e_{1}, v}^{\mathrm{NE}} \vee G_{u+e_{2}, v}^{\mathrm{NE}} \quad \text { for } u \leq v-e_{1}-e_{2} .
\end{aligned}
$$

It is immediate from recovery $\omega_{x}=B\left(x, x+e_{1}\right) \wedge B\left(x, x+e_{2}\right)$ and additivity of $B$ that

$$
G_{u, v}^{\mathrm{NE}}=B(u, v) \text { for all } u \leq v
$$

Process $\left\{G_{u, v}^{\mathrm{NE}}: u \leq v\right\}$ is stationary in the sense that the increments

$$
G_{x, v}^{\mathrm{NE}}-G_{x+e_{i}, v}^{\mathrm{NE}}=B\left(x, x+e_{i}\right)
$$

are stationary under lattice translations and, as the equation above reveals, do not depend on the choice of the origin $v$ (as long as we stay southwest of the origin).

Remark 5.5 In the exactly solvable cases where $\omega_{x}$ is either exponential or geometric, more is known. Given the stationary cocycle, define weights

$$
Y_{x}=B\left(x-e_{1}, x\right) \wedge B\left(x-e_{2}, x\right) .
$$

Then the weights $\left\{Y_{x}\right\}$ have the same i.i.d. distribution as the original weights $\left\{\omega_{x}\right\}$. Furthermore, $\left\{Y_{x}: x \geq v+e_{1}+e_{2}\right\}$ are independent of $\left\{B\left(v+k e_{i}, v+(k+1) e_{i}\right): k \in\right.$ $\left.\mathbb{Z}_{+}, i \in\{1,2\}\right\}$. Hence a stationary last-passage percolation process can be defined in 
the first quadrant with cocycles on the south and west boundaries:

$$
\begin{aligned}
G_{v, x}^{\mathrm{SW}} & =B(v, x) \text { for } x \in\left\{v+k e_{i}: k \in \mathbb{Z}_{+}, i \in\{1,2\}\right\} \\
\text { and } \quad G_{v, x}^{\mathrm{SW}} & =Y_{x}+G_{v, x-e_{1}}^{\mathrm{SW}} \vee G_{v, x-e_{2}}^{\mathrm{SW}} \text { for } x \geq v+e_{1}+e_{2} .
\end{aligned}
$$

This feature appears in [10] as the "Burke property" of the exponential last-passage model. It also works for the log-gamma polymer in positive temperature $[28,55]$. We do not know presently if this works in the general last-passage case.

\subsection{Solution to the variational formulas}

In this section we construct minimizers for variational formulas (2.14)-(2.15) in terms of the cocycles from Theorem 5.2. The theorems below are versions of Theorem 3.2 that we can state without extra regularity assumptions on the shape function $g_{\mathrm{pp}}$. The proof of Theorem 3.2 has to wait till Sect. 6, where we identify the minimizing cocycles used below as Busemann functions under regularity assumptions on $g_{\mathrm{pp}}$.

Recall from Theorem 4.4 that $g_{\mathrm{pp}}$ is linear over each line segment $[\xi(h), \bar{\xi}(h)]$ and hence, by Theorem 5.2(iii), cocycles $B^{\xi}$ coincide for all $\left.\xi \in\right] \underline{\xi}(h), \bar{\xi}(h)[$.

Theorem 5.6 Let $\left\{B_{ \pm}^{\xi}\right\}$ be the cocycles given in Theorem 5.2. Fix $h \in \mathbb{R}^{2}$. Let $t(h)$, $\xi(h)$, and $\bar{\xi}(h)$ be as in Theorem 4.4. One has the following three cases.

(i) $\underline{\xi}(h) \neq \bar{\xi}(h):$ For any (and hence all) $\xi \in] \underline{\xi}(h), \bar{\xi}(h)[$ let

$$
F^{h}(\widehat{\omega}, x, y)=h(\xi) \cdot(x-y)-B^{\xi}(\widehat{\omega}, x, y)
$$

Then for $\widehat{\mathbb{P}}$-almost every $\widehat{\omega}$

$$
g_{\mathrm{pl}}(h)=\max _{i=1,2}\left\{\omega_{0}+h \cdot e_{i}+F^{h}\left(\widehat{\omega}, 0, e_{i}\right)\right\}=t(h) .
$$

(ii) $\underline{\xi}(h)=\bar{\xi}(h)=\xi \in \mathscr{D}$ : (5.10) holds for $F^{h}$ defined as in (5.9).

(iii) $\underline{\xi}(h)=\bar{\xi}(h)=\xi \notin \mathscr{D}$ : Let $\theta \in[0,1]$ be such that

$$
h-t(h)\left(e_{1}+e_{2}\right)=\theta h_{-}(\xi)+(1-\theta) h_{+}(\xi)
$$

and define

$$
\begin{aligned}
F^{\xi \pm}(\widehat{\omega}, x, y) & =h_{ \pm}(\xi) \cdot(x-y)-B_{ \pm}^{\xi}(\widehat{\omega}, x, y) \text { and } \\
F^{h}(\widehat{\omega}, x, y) & =\theta F^{\xi-}(\widehat{\omega}, x, y)+(1-\theta) F^{\xi+}(\widehat{\omega}, x, y) .
\end{aligned}
$$


If $\theta \in\{0,1\},(5.10)$ holds again almost surely. For all $\theta \in[0,1]$ we have

$$
\begin{aligned}
& g_{\mathrm{pl}}(h)=\mathbb{P} \text {-ess sup } \max _{i=1,2}\left\{\omega_{0}+h \cdot e_{i}+\widehat{\mathbb{E}}\left[F^{h}\left(0, e_{i}\right) \mid \mathfrak{S}\right]\right\} \\
& =\widehat{\mathbb{P}} \text {-ess sup } \max _{i=1,2}\left\{\omega_{0}+h \cdot e_{i}+F^{h}\left(\widehat{\omega}, 0, e_{i}\right)\right\}=t(h) .
\end{aligned}
$$

In particular, in all cases $(i)-\left(\right.$ iii), $\widehat{\mathbb{E}}\left[F^{h}(x, y) \mid \mathfrak{S}\right] \in \mathscr{K}_{0}(\Omega)$ is a minimizer in (2.14).

Here are qualitative descriptions of the cases above.

(i) The graph of $f$ has a corner at the point $(\alpha, f(\alpha))$ where it crosses the $45^{\circ}$ line through $-h$. Correspondingly, $g_{\mathrm{pp}}$ is linear on $[\underline{\xi}(h), \bar{\xi}(h)]$ with gradient $\nabla g_{\mathrm{pp}}(\xi)=(\alpha, f(\alpha))$ at interior points $\left.\xi \in\right] \underline{\xi}(h), \bar{\xi}(h)[$.

(ii) The unique $\xi$ dual to $h$ lies in $\mathscr{E}$.

(iii) The unique $\xi$ dual to $h$ is exposed but not in $\mathscr{D}$.

Proof of Theorem 5.6 Let $B$ be one of $B^{\xi}, B_{ \pm}^{\xi}$ and define centered cocycle $F$ by (2.13). By (5.3) and (4.16), $g_{\mathrm{pl}}(h(B))=0$. Then directly from definitions (2.2) and (2.4), $g_{\mathrm{pl}}(h)=(h-h(B)) \cdot e_{j}$ for $j \in\{1,2\}$ for any $h \in \mathbb{R}^{2}$ that satisfies $(h-h(B)) \cdot\left(e_{2}-\right.$ $\left.e_{1}\right)=0$. Hence by recovery (5.2), for these same $h$-values, for $\widehat{\mathbb{P}}$-a.e. $\widehat{\omega}$ and $j \in\{1,2\}$,

$$
g_{\mathrm{pl}}(h)=\max _{i \in\{1,2\}}\left\{\omega_{0}+h \cdot e_{i}+F\left(\widehat{\omega}, 0, e_{i}\right)\right\}=(h-h(B)) \cdot e_{j} .
$$

(This situation is developed for general models in Theorem 3.4 of [27].)

In cases (i) and (ii), (5.10) comes from (5.12) combined with (4.14) and (5.3). The same works in case (iii) when $\theta \in\{0,1\}$.

Consider case (iii). Using Theorem 4.4 and $g_{\mathrm{pl}}\left(h_{ \pm}(\xi)\right)=0$ gives

$$
\begin{aligned}
g_{\mathrm{pl}}(h) & =g_{\mathrm{pp}}(\xi)+h \cdot \xi \\
& =t(h)+\theta\left(g_{\mathrm{pp}}(\xi)+h_{-}(\xi) \cdot \xi\right)+(1-\theta)\left(g_{\mathrm{pp}}(\xi)+h_{+}(\xi) \cdot \xi\right) \\
& =t(h)+\theta g_{\mathrm{pl}}\left(h_{-}(\xi)\right)+(1-\theta) g_{\mathrm{pl}}\left(h_{+}(\xi)\right)=t(h) .
\end{aligned}
$$

By (5.2), $\widehat{\mathbb{P}}$-almost surely

$$
\min \left\{\theta B_{-}^{\xi}\left(0, e_{1}\right)+(1-\theta) B_{+}^{\xi}\left(0, e_{1}\right), \theta B_{-}^{\xi}\left(0, e_{2}\right)+(1-\theta) B_{+}^{\xi}\left(0, e_{2}\right)\right\} \geq \omega_{0} .
$$

Since $\widehat{\mathbb{E}}\left[F^{h}(x, y) \mid \mathfrak{S}\right]$ is a member of $\mathscr{K}_{0}(\Omega)$, we can use (2.14) to verify (5.11):

$$
\begin{aligned}
g_{\mathrm{pl}}(h) & \leq \mathbb{P} \text {-ess sup } \max _{i=1,2}\left\{\omega_{0}+h \cdot e_{i}+\widehat{\mathbb{E}}\left[F^{h}\left(0, e_{i}\right) \mid \mathfrak{S}\right]\right\} \\
& \leq \widehat{\mathbb{P}} \text {-ess sup } \max _{\widehat{\omega}=1,2}\left\{\omega_{0}+h \cdot e_{i}+F^{h}\left(\widehat{\omega}, 0, e_{i}\right)\right\} \leq t(h)=g_{\mathrm{pl}}(h) .
\end{aligned}
$$

The last inequality above is just a rearrangement of (5.13). The same inequalities show that $\widehat{\mathbb{E}}\left[F^{h}\left(0, e_{i}\right) \mid \mathfrak{S}\right]$ minimizes in (2.14) in cases (i) and (ii). 
We state also the corresponding theorem for the point-to-point case. Using the duality (2.7) of $h_{ \pm}(\xi)$ and $\xi$, it follows as the theorem above.

Theorem 5.7 Let $\xi \in \operatorname{ri} \mathscr{U}$. Then for $\widehat{\mathbb{P}}$-a.e. $\widehat{\omega}$

$$
g_{\mathrm{pp}}(\xi)=\max _{i=1,2}\left\{\omega_{0}-B_{ \pm}^{\xi}\left(\widehat{\omega}, 0, e_{i}\right)-h_{ \pm}(\xi) \cdot \xi\right\}=-h_{ \pm}(\xi) \cdot \xi
$$

For any $\theta \in[0,1]$, cocycle $\widehat{\mathbb{E}}\left[\theta B_{-}^{\xi}+(1-\theta) B_{+}^{\xi} \mid \mathfrak{S}\right] \in \mathscr{K}(\Omega)$ is a minimizer in $(2.15)$.

\section{Busemann functions from cocycles}

In this section we prove the existence of Busemann functions. As before, (2.1) is a standing assumption. No regularity assumptions are made on $g_{\mathrm{pp}}$, hence the results below are in terms of inequalities for limsup and liminf. Under additional regularity assumptions, the sharper theorems claimed in earlier sections are derived as corollaries.

Recall the line segment $\mathscr{U} \xi=[\underline{\xi}, \bar{\xi}]$ with $\underline{\xi} \cdot e_{1} \leq \bar{\xi} \cdot e_{1}$ from (2.10)-(2.11) and the cocycles $B_{ \pm}^{\xi}$ constructed on the extended space $(\widehat{\Omega}, \widehat{S}, \widehat{\mathbb{P}})$ in Theorem 5.2.

Theorem 6.1 Fix a possibly degenerate segment $[\zeta, \eta] \subset$ ri $\mathscr{U}$. Assume either that there is an exposed point $\xi$ such that $\xi=\underline{\xi}=\bar{\xi}$ and $[\zeta, \eta]=\{\xi\}=[\underline{\xi}, \bar{\xi}]$, or that $[\zeta, \eta]$ a maximal nondegenerate linear segment of $g_{\mathrm{pp}}$ in which case $[\zeta, \eta]=[\xi, \bar{\xi}]$ for any $\xi \in] \zeta, \eta\left[\right.$. Then there exists an event $\widehat{\Omega}_{0}$ with $\widehat{\mathbb{P}}\left(\widehat{\Omega}_{0}\right)=1$ such that for each $\widehat{\omega} \in \widehat{\Omega}_{0}$ and for any sequence $v_{n} \in \mathbb{Z}_{+}^{2}$ that satisfies

$$
\left|v_{n}\right|_{1} \rightarrow \infty \text { and } \underline{\xi} \cdot e_{1} \leq \lim _{n \rightarrow \infty} \frac{v_{n} \cdot e_{1}}{\left|v_{n}\right|_{1}} \leq \varlimsup_{n \rightarrow \infty} \frac{v_{n} \cdot e_{1}}{\left|v_{n}\right|_{1}} \leq \bar{\xi} \cdot e_{1}
$$

we have for all $x \in \mathbb{Z}^{2}$

$$
\begin{aligned}
B_{+}^{\bar{\xi}}\left(\widehat{\omega}, x, x+e_{1}\right) & \leq \varliminf_{n \rightarrow \infty}\left(G_{x, v_{n}}(\omega)-G_{x+e_{1}, v_{n}}(\omega)\right) \\
& \leq \varlimsup_{n \rightarrow \infty}\left(G_{x, v_{n}}(\omega)-G_{x+e_{1}, v_{n}}(\omega)\right) \leq B_{-}^{\underline{\xi}}\left(\widehat{\omega}, x, x+e_{1}\right)
\end{aligned}
$$

and

$$
\begin{aligned}
B_{-}^{\underline{\xi}}\left(\widehat{\omega}, x, x+e_{2}\right) & \leq \varliminf_{n \rightarrow \infty}\left(G_{x, v_{n}}(\omega)-G_{x+e_{2}, v_{n}}(\omega)\right) \\
& \leq \varlimsup_{n \rightarrow \infty}\left(G_{x, v_{n}}(\omega)-G_{x+e_{2}, v_{n}}(\omega)\right) \leq B_{+}^{\bar{\xi}}\left(\widehat{\omega}, x, x+e_{2}\right) .
\end{aligned}
$$

Let us emphasize that the sequence $v_{n}$ is allowed to depend on $\widehat{\omega} \in \widehat{\Omega}_{0}$. The interesting cases are of course the ones where we have a limit. The next corollary follows immediately because if $\xi, \underline{\xi}, \bar{\xi} \in \mathscr{D}$ then by Theorem 5.2(iii) $B_{ \pm}^{\xi}=B^{\xi}=B_{ \pm}^{\bar{\xi}}$ for all $\xi \in[\underline{\xi}, \bar{\xi}]$. 
Corollary 6.2 Assume $\xi, \xi, \bar{\xi} \in \mathscr{D}$. Then there exists an event $\widehat{\Omega}_{0}$ with $\widehat{\mathbb{P}}\left(\widehat{\Omega}_{0}\right)=1$ such that for each $\widehat{\omega} \in \Omega_{0}$, for any sequence $v_{n} \in \mathbb{Z}_{+}^{2}$ that satisfies (6.1), and for all $x, y \in \mathbb{Z}^{2}$,

$$
B^{\xi}(\widehat{\omega}, x, y)=\lim _{n \rightarrow \infty}\left(G_{x, v_{n}}(\omega)-G_{y, v_{n}}(\omega)\right) .
$$

The limit implies that $\mathbb{P}$-a.s. $B^{\xi}(\widehat{\omega}, x, y)$ is a function of $(\omega, x, y)$.

In particular, if $g_{\mathrm{pp}}$ is differentiable everywhere on ri $\mathscr{U}$, then for each direction $\xi \in \mathrm{ri} \mathscr{U}$ there is an event of full $\widehat{\mathbb{P}}$-probability on which limit (6.4) holds for any sequence $v_{n} /\left|v_{n}\right|_{1} \rightarrow \xi$ with $\left|v_{n}\right|_{1} \rightarrow \infty$.

Before the proof of Theorem 6.1, we complete the proofs of some earlier theorems.

Proof of Theorem 3.1 Limit (3.2) is in Corollary 6.2. Equation (3.4) follows from (5.3).

To prove the point-to-line limit (3.3) recall the duality from Theorem 4.4. Observe that

$$
\begin{aligned}
\zeta \in[\underline{\xi}, \bar{\xi}] & \Longleftrightarrow g_{\mathrm{pp}}(\zeta)=g_{\mathrm{pp}}(\xi)+\nabla g_{\mathrm{pp}}(\xi) \cdot(\zeta-\xi) \\
& \Longleftrightarrow g_{\mathrm{pp}}(\zeta)=g_{\mathrm{pp}}(\xi)-h \cdot(\zeta-\xi) \Longleftrightarrow \zeta \in[\underline{\xi}(h), \bar{\xi}(h)] .
\end{aligned}
$$

Let $z \in\left\{e_{1}, e_{2}\right\}$. Pick ( $\omega$-dependent) $u_{n}, v_{n} \in \mathbb{Z}_{+}^{2}$ so that $\left|u_{n}\right|_{1}=\left|v_{n}\right|_{1}=n$ and

$$
G_{n}(h)=G_{0, u_{n}}+h \cdot u_{n} \text { and } G_{n-1}(h) \circ T_{z}=G_{z, v_{n}}+h \cdot\left(v_{n}-z\right) .
$$

Fix $\omega$ so that limits (2.4) and (2.5) hold both for $\omega$ and $T_{z} \omega$. Then taking $n \rightarrow \infty$ along suitable subsequences shows that all limit points of $u_{n}(\omega) / n$ and $v_{n}(\omega) / n$ satisfy duality (2.7) and so lie in $[\underline{\xi}(h), \bar{\xi}(h)]$. By (6.5) these sequences satisfy (6.1). Let $n \rightarrow \infty$ in the inequalities

$$
G_{0, v_{n}}-G_{z, v_{n}}+h \cdot z \leq G_{n}(h)-G_{n-1}(h) \circ T_{z} \leq G_{0, u_{n}}-G_{z, u_{n}}+h \cdot z,
$$

and use (6.4) to get the conclusion.

Proof of Theorem 3.2 The theorem follows from Theorems 5.6 and 5.7 because the Busemann function $B^{\xi}$ is the cocycle $B^{\xi}$ from Theorem 5.2.

Proof of Theorem 5.3 Under the assumption of differentiability at endpoints of linear segments, every $\xi \in \mathscr{D}$ satisfies Corollary 6.2 and so $B^{\xi}(\cdot, x, y)$ is $\mathfrak{S}$-measurable. Any other point $\zeta \in$ ri $\mathscr{U}$ is a limit from both left and right of $\mathscr{D}$-points, and so by parts (iv) and (v) of Theorem 5.2, cocycles $B_{ \pm}^{\zeta}$ are a.s. limits of S-measurable cocycles.

The remainder of this section proves Theorem 6.1. We begin with a general comparison lemma. This idea goes back at least to $[1,2]$. With arbitrary real weights $\left\{\widetilde{Y}_{x}\right\}_{x \in \mathbb{Z}^{2}}$ define last-passage times

$$
\widetilde{G}_{u, v}=\max _{x_{0, n}} \sum_{k=0}^{n-1} \widetilde{Y}_{x_{k}} .
$$


The maximum is over up-right paths from $x_{0}=u$ to $x_{n}=v$ with $n=|v-u|_{1}$. The convention is $\widetilde{G}_{v, v}=0$. For $x \leq v-e_{1}$ and $y \leq v-e_{2}$ denote the increments by

$$
\widetilde{I}_{x, v}=\widetilde{G}_{x, v}-\widetilde{G}_{x+e_{1}, v} \quad \text { and } \quad \widetilde{J}_{y, v}=\widetilde{G}_{y, v}-\widetilde{G}_{y+e_{2}, v} .
$$

Lemma 6.3 For $x \leq v-e_{1}$ and $y \leq v-e_{2}$

$$
\widetilde{I}_{x, v+e_{2}} \geq \widetilde{I}_{x, v} \geq \widetilde{I}_{x, v+e_{1}} \quad \text { and } \quad \widetilde{J}_{y, v+e_{2}} \leq \widetilde{J}_{y, v} \leq \widetilde{J}_{y, v+e_{1}}
$$

Proof Let $v=(m, n)$. The proof goes by an induction argument. Suppose $x=(k, n)$ for some $k<m$. Then on the north boundary

$$
\begin{aligned}
\widetilde{I}_{(k, n),(m, n+1)} & =\widetilde{G}_{(k, n),(m, n+1)}-\widetilde{G}_{(k+1, n),(m, n+1)} \\
& =\widetilde{Y}_{k, n}+\widetilde{G}_{(k+1, n),(m, n+1)} \vee \widetilde{G}_{(k, n+1),(m, n+1)}-\widetilde{G}_{(k+1, n),(m, n+1)} \\
& \geq \widetilde{Y}_{k, n}=\widetilde{G}_{(k, n),(m, n)}-\widetilde{G}_{(k+1, n),(m, n)}=\widetilde{I}_{(k, n),(m, n)} .
\end{aligned}
$$

On the east boundary, when $y=(m, \ell)$ for some $\ell<n$

$$
\begin{aligned}
\widetilde{J}_{(m, \ell),(m, n+1)} & =\widetilde{G}_{(m, \ell),(m, n+1)}-\widetilde{G}_{(m, \ell+1),(m, n+1)} \\
& =\widetilde{Y}_{m, \ell}=\widetilde{G}_{(m, \ell),(m, n)}-\widetilde{G}_{(m, \ell+1),(m, n)}=\widetilde{J}_{(m, \ell),(m, n)} .
\end{aligned}
$$

These inequalities start the induction. Now let $u \leq v-e_{1}-e_{2}$. Assume by induction that (6.6) holds for $x=u+e_{2}$ and $y=u+e_{1}$.

$$
\begin{aligned}
\widetilde{I}_{u, v+e_{2}} & =\widetilde{G}_{u, v+e_{2}}-\widetilde{G}_{u+e_{1}, v+e_{2}}=\widetilde{Y}_{u}+\left(\widetilde{G}_{u+e_{2}, v+e_{2}}-\widetilde{G}_{u+e_{1}, v+e_{2}}\right)^{+} \\
& =\widetilde{Y}_{u}+\left(\widetilde{I}_{u+e_{2}, v+e_{2}}-\widetilde{J}_{u+e_{1}, v+e_{2}}\right)^{+} \\
& \geq \widetilde{Y}_{u}+\left(\widetilde{I}_{u+e_{2}, v}-\widetilde{J}_{u+e_{1}, v}\right)^{+}=\widetilde{I}_{u, v}
\end{aligned}
$$

The last equality comes by repeating the first three equalities with $v$ instead of $v+e_{2}$. A similar argument works for $\widetilde{I}_{u, v} \geq \widetilde{I}_{u, v+e_{1}}$ and a symmetric argument works for the $\widetilde{J}$ inequalities.

The estimates needed for the proof of Theorem 6.1 come from coupling $G_{u, v}$ with the stationary LPP described in Sect. 5.2. For the next two lemmas fix $\zeta \in$ ri $\mathscr{U}$ and a cocycle $B(\widehat{\omega}, x, y)=B_{ \pm}^{\zeta}(\widehat{\omega}, x, y)$ from Theorem 5.2. Let $r=\zeta \cdot e_{1} / \zeta \cdot e_{2}$ so that $\alpha=\gamma^{\prime}(r \pm)$ satisfies

$$
\alpha=\widehat{\mathbb{E}}\left[B\left(x, x+e_{1}\right)\right] \text { and } f(\alpha)=\widehat{\mathbb{E}}\left[B\left(x, x+e_{2}\right)\right] .
$$

As in (5.7) define

$$
\begin{aligned}
G_{u, v}^{\mathrm{NE}} & =B(u, v) \quad \text { for } u \in\left\{v-k e_{i}: k \in \mathbb{Z}_{+}, i \in\{1,2\}\right\} \\
\text { and } \quad G_{u, v}^{\mathrm{NE}} & =\omega_{u}+G_{u+e_{1}, v}^{\mathrm{NE}} \vee G_{u+e_{2}, v}^{\mathrm{NE}} \quad \text { for } u \leq v-e_{1}-e_{2} .
\end{aligned}
$$


Fig. 3 Illustration of (6.9).

Forcing the last step to be $e_{1}$ restricts the maximization to paths that hit the north boundary instead of the east boundary. The path from 0 to $(s-\tau, t)$ contributes $g_{\mathrm{pp}}(s-\tau, t)$ and the remaining segment of length $\tau$ on the north boundary contributes $\alpha \tau$

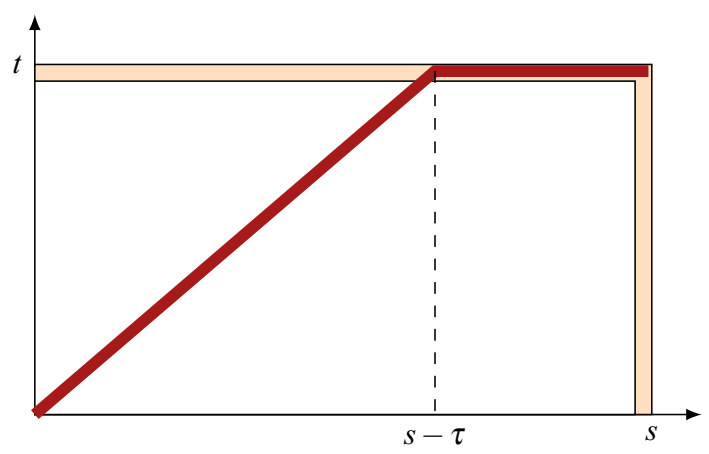

Let $G_{u, v}^{\mathrm{NE}}(A)$ denote a maximum over paths restricted to the set $A$. In particular, below we use

$$
G_{0, v}^{\mathrm{NE}}\left(v-e_{i} \in x_{.}\right)=\max _{x_{*}: x_{|v|_{1}-1}=v-e_{i}} \sum_{k=0}^{|v|_{1}-1} \widetilde{Y}_{x_{k}}
$$

where the maximum is restricted to paths that go through the point $v-e_{i}$, and the weights are from (6.8): $\widetilde{Y}_{x}=\omega_{x}$ for $x \leq v-e_{1}-e_{2}$ while $\widetilde{Y}_{v-k e_{i}}=B\left(v-k e_{i}, v-\right.$ $\left.(k-1) e_{i}\right)$.

Figure 3 makes the limits of the next lemma obvious. But a.s. convergence requires some technicalities because the north-east boundaries themselves are translated as the limit is taken.

Lemma 6.4 Assume (2.1). Fix reals $0<s, t<\infty$. Let $v_{n} \in \mathbb{Z}_{+}^{2}$ be such that $v_{n} /\left|v_{n}\right|_{1} \rightarrow(s, t) /(s+t)$ as $n \rightarrow \infty$ and $\left|v_{n}\right|_{1} \geq \eta_{0} n$ for some constant $\eta_{0}>0$. Then we have the following almost sure limits:

$$
\left|v_{n}\right|_{1}^{-1} G_{0, v_{n}}^{\mathrm{NE}}\left(v_{n}-e_{1} \in x_{.}\right) \underset{n \rightarrow \infty}{\longrightarrow}(s+t)^{-1} \sup _{0 \leq \tau \leq s}\left\{\alpha \tau+g_{\mathrm{pp}}(s-\tau, t)\right\}
$$

and

$$
\left|v_{n}\right|_{1}^{-1} G_{0, v_{n}}^{\mathrm{NE}}\left(v_{n}-e_{2} \in x_{*}\right) \underset{n \rightarrow \infty}{\longrightarrow}(s+t)^{-1} \sup _{0 \leq \tau \leq t}\left\{f(\alpha) \tau+g_{\mathrm{pp}}(s, t-\tau)\right\} .
$$

Proof We prove (6.9). Fix $\varepsilon>0$, let $M=\left\lfloor\varepsilon^{-1}\right\rfloor$, and

$$
q_{j}^{n}=j\left\lfloor\frac{\varepsilon\left|v_{n}\right|_{1} s}{s+t}\right\rfloor \text { for } 0 \leq j \leq M-1 \text {, and } q_{M}^{n}=v_{n} \cdot e_{1} .
$$

For large enough $n$ it is the case that $q_{M-1}^{n}<v_{n} \cdot e_{1}$. 
Suppose a maximal path for $G_{0, v_{n}}^{\mathrm{NE}}\left(v_{n}-e_{1} \in x_{\text {. }}\right)$ enters the north boundary from the bulk at the point $v_{n}-(\ell, 0)$ with $q_{j}^{n}<\ell \leq q_{j+1}^{n}$. By superadditivity

$$
\begin{aligned}
& G_{0, v_{n}}^{\mathrm{NE}}\left(v_{n}-e_{1} \in x_{.}\right)=G_{0, v_{n}-(\ell, 1)}+\omega_{v_{n}-(\ell, 1)}+B\left(v_{n}-(\ell, 0), v_{n}\right) \\
& \leq G_{0, v_{n}-\left(q_{j}^{n}, 1\right)}+q_{j}^{n} \alpha-\sum_{k=q_{j}^{n}+1}^{\ell-1}\left(\omega_{v_{n}-(k, 1)}-m_{0}\right)+\left(\ell-1-q_{j}^{n}\right) m_{0} \\
& \quad+\left(B\left(v_{n}-(\ell, 0), v_{n}\right)-\ell \alpha\right)+\left(\ell-q_{j}^{n}\right) \alpha .
\end{aligned}
$$

The two main terms come right after the inequality above and the rest are errors.

Define the centered cocycle $F(x, y)=h(B) \cdot(x-y)-B(x, y)$ so that

$$
B\left(v_{n}-(\ell, 0), v_{n}\right)-\ell \alpha=F\left(0, v_{n}-(\ell, 0)\right)-F\left(0, v_{n}\right)
$$

The potential-recovery property $(5.1) \omega_{0}=B\left(0, e_{1}\right) \wedge B\left(0, e_{2}\right)$ gives

$$
F\left(0, e_{i}\right) \leq \alpha \vee f(\alpha)-\omega_{0} \quad \text { for } i \in\{1,2\}
$$

The i.i.d. distribution of $\left\{\omega_{x}\right\}$ and $\mathbb{E}\left(\left|\omega_{0}\right|^{p}\right)<\infty$ with $p>2$ are strong enough to guarantee that Lemma 7.8 from Appendix applies and gives

$$
\lim _{N \rightarrow \infty} \frac{1}{N} \max _{x \geq 0:|x|_{1} \leq N}|F(\widehat{\omega}, 0, x)|=0 \quad \text { for a.e. } \widehat{\omega} \text {. }
$$

Collect the bounds for all the intervals $\left(q_{j}^{n}, q_{j+1}^{n}\right]$ and let $C$ denote a constant. Abbreviate $S_{j, m}^{n}=\sum_{k=q_{j}^{n}+1}^{q_{j}^{n}+m}\left(\omega_{v_{n}-(k, 1)}-m_{0}\right)$.

$$
\begin{aligned}
G_{0, v_{n}}^{\mathrm{NE}}\left(v_{n}-e_{1} \in x_{\bullet}\right) \leq & \max _{0 \leq j \leq M-1}\left\{G_{0, v_{n}-\left(q_{j}^{n}, 1\right)}+q_{j}^{n} \alpha+C\left(q_{j+1}^{n}-q_{j}^{n}\right)\right. \\
& \left.+\max _{0 \leq m<q_{j+1}^{n}-q_{j}^{n}}\left|S_{j, m}^{n}\right|+\max _{q_{j}^{n}<\ell \leq q_{j+1}^{n}} F\left(0, v_{n}-(\ell, 0)\right)-F\left(0, v_{n}\right)\right\}
\end{aligned}
$$

Divide through by $\left|v_{n}\right|_{1}$ and let $n \rightarrow \infty$. Limit (2.5) gives convergence of the $G$-term on the right. We claim that the terms on the second line of (6.12) vanish. Limit (6.11) takes care of the $F$-terms. Combine Doob's maximal inequality for martingales with Burkholder's inequality [11, Thm. 3.2] to obtain, for $\delta>0$,

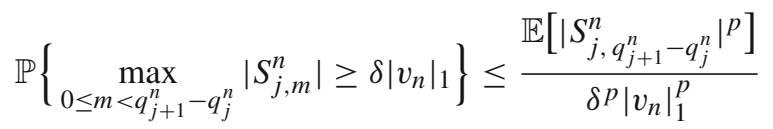

$$
\begin{aligned}
& \leq \frac{C}{\delta^{p}\left|v_{n}\right|_{1}^{p}} \mathbb{E}\left[\left|\sum_{i=1}^{q_{j+1}^{n}-q_{j}^{n}}\left(\omega_{i, 0}-m_{0}\right)^{2}\right|^{p / 2}\right] \leq \frac{C}{\left|v_{n}\right|_{1}^{p / 2}} .
\end{aligned}
$$


Thus Borel-Cantelli takes care of the $S_{j, m}^{n}$-term on the second line of (6.12). (This is the place where the assumption $\left|v_{n}\right|_{1} \geq \eta_{0} n$ is used.) We have the upper bound

$\varlimsup_{n \rightarrow \infty}\left|v_{n}\right|_{1}^{-1} G_{0, v_{n}}^{\mathrm{NE}}\left(v_{n}-e_{1} \in x_{.}\right) \leq(s+t)^{-1} \max _{0 \leq j \leq M-1}\left[g_{\mathrm{pp}}(s-s j \varepsilon, t)+s j \varepsilon \alpha+C \varepsilon s\right]$.

Let $\varepsilon \searrow 0$ to complete the proof of the upper bound.

To get the matching lower bound let the supremum $\sup _{\tau \in[0, s]}\left\{\tau \alpha+g_{\mathrm{pp}}(s-\tau, t)\right\}$ be attained at $\tau^{*} \in[0, s]$. With $m_{n}=\left|v_{n}\right|_{1} /(s+t)$ we have

$$
\begin{aligned}
G_{0, v_{n}}^{\mathrm{NE}}\left(v_{n}-e_{1} \in x_{.}\right) \geq & G_{0, v_{n}-\left(\left\lfloor m_{n} \tau^{*}\right\rfloor \vee 1,1\right)}+\omega_{v_{n}-\left(\left\lfloor m_{n} \tau^{*}\right\rfloor \vee 1,1\right)} \\
& +B\left(v_{n}-\left(\left\lfloor m_{n} \tau^{*}\right\rfloor \vee 1,0\right), v_{n}\right) .
\end{aligned}
$$

Use again the cocycle $F$ from above, and let $n \rightarrow \infty$ to get

$$
\varliminf_{n \rightarrow \infty}\left|v_{n}\right|_{1}^{-1} G_{0, v_{n}}^{\mathrm{NE}}\left(v_{n}-e_{1} \in x_{.}\right) \geq(s+t)^{-1}\left[g_{\mathrm{pp}}\left(s-\tau^{*}, t\right)+\tau^{*} \alpha\right] .
$$

This completes the proof of (6.9).

Continue with the stationary LPP defined by (6.8) in terms of a cocycle $B=B_{ \pm}^{\zeta}$, with $r=\zeta \cdot e_{1} / \zeta \cdot e_{2}$ and $\alpha$ as in (6.7). Let us call the direction $\zeta$ characteristic for $\alpha$. The next lemma shows that in stationary LPP a maximizing path to a point below the characteristic direction will eventually hit the north boundary before the east boundary. (Illustration in Fig. 4.) We omit the entirely analogous result and proof for a point above the characteristic line.

Lemma 6.5 Let $s \in(r, \infty)$. Let $v_{n} \in \mathbb{Z}_{+}^{2}$ be such that $v_{n} /\left|v_{n}\right|_{1} \rightarrow(s, 1) /(1+s)$ and $\left|v_{n}\right|_{1} \geq \eta_{0} n$ for some constant $\eta_{0}>0$. Assume that $\gamma^{\prime}(r+)>\gamma^{\prime}(s-)$. Then $\widehat{\mathbb{P}}-$ a.s.

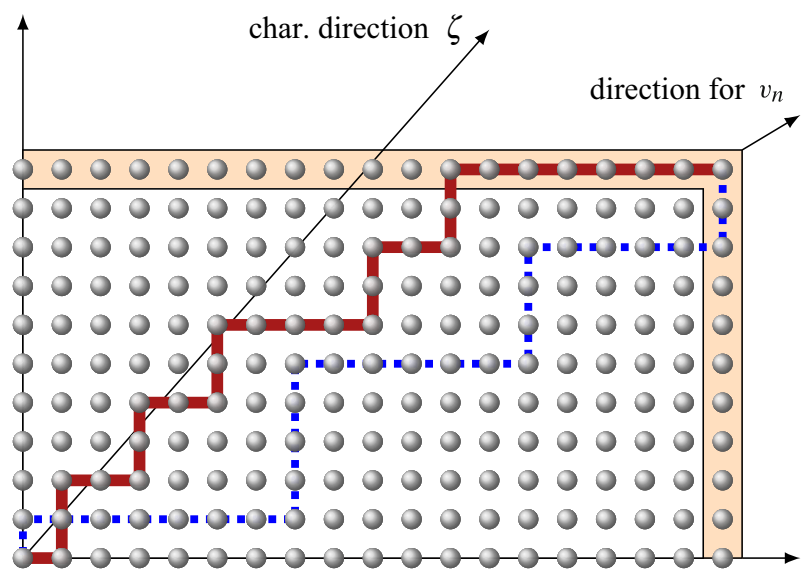

Fig. 4 Illustration of Lemma 6.5. With $\alpha$-boundaries geodesics tend to go in the $\alpha$-characteristic direction $\zeta$. If $v_{n}$ converges in a direction below $\zeta$, maximal paths to $v_{n}$ tend to hit the north boundary. The dotted path that hits the east boundary is unlikely to be maximal for large $n$ 
there exists a random $n_{0}<\infty$ such that for all $n \geq n_{0}$,

$$
G_{0, v_{n}}^{\mathrm{NE}}=G_{0, v_{n}}^{\mathrm{NE}}\left(v_{n}-e_{1} \in x_{.}\right)
$$

Proof The right derivative at $\tau=0$ of $\alpha \tau+g_{\mathrm{pp}}(s-\tau, 1)=\alpha \tau+\gamma(s-\tau)$ equals

$$
\alpha-\gamma^{\prime}(s-)>\alpha-\gamma^{\prime}(r+) \geq 0 \text {. }
$$

The last inequality above follows from the assumption on $r$. Thus we can find $\tau^{*} \in$ $(0, r)$ such that

$$
\alpha \tau^{*}+g_{\mathrm{pp}}\left(s-\tau^{*}, 1\right)>g_{\mathrm{pp}}(s, 1) .
$$

To produce a contradiction let $A$ be the event on which $G_{0, v_{n}}^{\mathrm{NE}}=G_{0, v_{n}}^{\mathrm{NE}}\left(v_{n}-e_{2} \in x_{\text {. }}\right)$ for infinitely many $n$ and assume $\widehat{\mathbb{P}}(A)>0$. Let $m_{n}=\left|v_{n}\right|_{1} /(1+s)$. On $A$ we have for infinitely many $n$

$$
\begin{aligned}
& \left|v_{n}\right|^{-1} G_{0, v_{n}}^{\mathrm{NE}}\left(v_{n}-e_{2} \in x_{*}\right)=\left|v_{n}\right|^{-1} G_{0, v_{n}}^{\mathrm{NE}} \\
& \quad \geq\left|v_{n}\right|^{-1} B\left(v_{n}-\left(\left\lfloor m_{n} \tau^{*}\right\rfloor+1\right) e_{1}, v_{n}\right)+\left|v_{n}\right|^{-1} G_{0, v_{n}-\left(\left\lfloor m_{n} \tau^{*}\right\rfloor+1,1\right)} \\
& \quad+\left|v_{n}\right|^{-1} \omega_{v_{n}-\left(\left\lfloor m_{n} \tau^{*}\right\rfloor+1,1\right) .}
\end{aligned}
$$

Apply (6.10) to the leftmost quantity. Apply limits (2.5) and (6.11) and stationarity and integrability of $\omega_{x}$ to the expression on the right. Both extremes of the above inequality converge almost surely. Hence on the event $A$ the inequality is preserved to the limit and yields (after multiplication by $1+s$ )

$$
\sup _{0 \leq \tau \leq 1}\left\{f(\alpha) \tau+g_{\mathrm{pp}}(s, 1-\tau)\right\} \geq \alpha \tau^{*}+g_{\mathrm{pp}}\left(s-\tau^{*}, 1\right) .
$$

The supremum of the left-hand side is achieved at $\tau=0$ because the right derivative equals

$$
f(\alpha)-\gamma^{\prime}\left(\frac{1-\tau}{s}-\right) \leq f(\alpha)-\gamma^{\prime}\left(r^{-1}-\right) \leq 0
$$

where the first inequality comes from $s^{-1}<r^{-1}$ and the second from (4.9). Therefore

$$
g_{\mathrm{pp}}(s, 1) \geq \alpha \tau^{*}+g_{\mathrm{pp}}\left(s-\tau^{*}, 1\right)
$$

which contradicts (6.14). Consequently $\widehat{\mathbb{P}}(A)=0$ and (6.13) holds for $n$ large.

Proof of Theorem 6.1 The proof goes in two steps.

Step 1. First consider a fixed $\xi=\left(\frac{s}{1+s}, \frac{1}{1+s}\right) \in$ ri $\mathscr{U}$ and a fixed sequence $v_{n}$ such that $v_{n} /\left|v_{n}\right|_{1} \rightarrow \xi$ and $\left|v_{n}\right|_{1} \geq \eta_{0} n$ for some $\eta_{0}>0$. We prove that the last inequality of (6.2) holds almost surely. Let $\zeta=\left(\frac{r}{1+r}, \frac{1}{1+r}\right)$ satisfy $\zeta \cdot e_{1}<\underline{\xi} \cdot e_{1}$ so that $\gamma^{\prime}(r+)>\gamma^{\prime}(s-)$ and Lemma 6.5 can be applied. Use cocycle $B^{\zeta+}$ from Theorem 
5.2 to define last-passage times $G_{u, v}^{\mathrm{NE}}$ as in (6.8). Furthermore, define last-passage times $G_{u, v}^{\mathrm{N}}$ that use cocycles only on the north boundary and bulk weights elsewhere:

$$
\begin{aligned}
& G_{v-k e_{1}, v}^{\mathrm{N}}=B^{\zeta+}\left(v-k e_{1}, v\right), \quad G_{v-\ell e_{2}, v}^{\mathrm{N}}=\sum_{j=1}^{\ell} \omega_{v-j e_{2}}, \\
& \quad \text { and } G_{u, v}^{\mathrm{N}}=\omega_{u}+G_{u+e_{1}, v}^{\mathrm{N}} \vee G_{u+e_{2}, v}^{\mathrm{N}} \quad \text { for } u \leq v-e_{1}-e_{2} .
\end{aligned}
$$

For large $n$ we have

$$
\begin{aligned}
G_{x, v_{n}}-G_{x+e_{1}, v_{n}} & \leq G_{x, v_{n}+e_{2}}^{\mathrm{N}}-G_{x+e_{1}, v_{n}+e_{2}}^{\mathrm{N}} \\
& =G_{x, v_{n}+e_{1}+e_{2}}^{\mathrm{NE}}\left(v_{n}+e_{2} \in x_{\bullet}\right)-G_{x+e_{1}, v_{n}+e_{1}+e_{2}}^{\mathrm{NE}}\left(v_{n}+e_{2} \in x_{\bullet}\right) \\
& =G_{x, v_{n}+e_{1}+e_{2}}^{\mathrm{NE}}-G_{x+e_{1}, v_{n}+e_{1}+e_{2}}^{\mathrm{NE}}=B_{+}^{\zeta}\left(x, x+e_{1}\right) .
\end{aligned}
$$

The first inequality above is the first inequality of (6.6). The first equality above is obvious. The second equality is Lemma 6.5 and the last equality is (5.8). Thus

$$
\varlimsup_{n \rightarrow \infty}\left(G_{x, v_{n}}-G_{x+e_{1}, v_{n}}\right) \leq B_{+}^{\zeta}\left(x, x+e_{1}\right) .
$$

Let $\zeta \cdot e_{1}$ increase to $\underline{\xi} \cdot e_{1}$. Theorem 5.2(iv) implies

$$
\varlimsup_{n \rightarrow \infty}\left(G_{x, v_{n}}-G_{x+e_{1}, v_{n}}\right) \leq B_{-}^{\underline{\xi}}\left(x, x+e_{1}\right) .
$$

An analogous argument gives the matching lower bound (first inequality of (6.2)) by taking $\zeta \cdot e_{1}>\bar{\xi} \cdot e_{1}$ and by reworking Lemma 6.5 for the case where the direction of $v_{n}$ is above the characteristic direction $\zeta$. Similar reasoning works for vertical increments $G_{x, v_{n}}-G_{x+e_{2}, v_{n}}$.

Step 2. We prove the full statement of Theorem 6.1. Let $\eta_{\ell}$ and $\zeta_{\ell}$ be two sequences in ri $\mathscr{U}$ such that $\eta_{\ell} \cdot e_{1}<\underline{\xi} \cdot e_{1} \leq \bar{\xi} \cdot e_{1}<\zeta_{\ell} \cdot e_{1}, \eta_{\ell} \rightarrow \underline{\xi}$, and $\zeta_{\ell} \rightarrow \bar{\xi}$. Let $\widehat{\Omega}_{0}$ be the event on which limits (5.5) hold for directions $\bar{\xi}$ and $\xi$ (with sequences $\zeta_{\ell}$ and $\eta_{\ell}$, respectively) and (6.2) holds for each direction $\zeta_{\ell}$ with sequence $\left\lfloor n \zeta_{\ell}\right\rfloor$, and for each direction $\eta_{\ell}$ with sequence $\left\lfloor n \eta_{\ell}\right\rfloor . \widehat{\mathbb{P}}\left(\widehat{\Omega}_{0}\right)=1$ by Theorem 5.2(iv) and Step 1 .

Fix any $\widehat{\omega} \in \widehat{\Omega}_{0}$ and a sequence $v_{n}$ as in (6.1). Abbreviate $a_{n}=\left|v_{n}\right|_{1}$. For each $\ell$ reason as follows. For large $n$

$$
\left\lfloor a_{n} \eta_{\ell}\right\rfloor \cdot e_{1}<v_{n} \cdot e_{1}<\left\lfloor a_{n} \zeta_{\ell}\right\rfloor \cdot e_{1} \text { and }\left\lfloor a_{n} \eta_{\ell}\right\rfloor \cdot e_{2}>v_{n} \cdot e_{2}>\left\lfloor a_{n} \zeta_{\ell}\right\rfloor \cdot e_{2} \text {. }
$$

By repeated application of the first inequality of (6.6),

$$
G_{x,\left\lfloor a_{n} \zeta_{\ell}\right\rfloor}-G_{x+e_{1},\left\lfloor a_{n} \zeta_{\ell}\right\rfloor} \leq G_{x, v_{n}}-G_{x+e_{1}, v_{n}} \leq G_{x,\left\lfloor a_{n} \eta_{\ell}\right\rfloor}-G_{x+e_{1},\left\lfloor a_{n} \eta_{\ell}\right\rfloor}
$$


Take $n \rightarrow \infty$ and apply (6.2) to the sequences $\left\lfloor a_{n} \zeta_{\ell}\right\rfloor$ and $\left\lfloor a_{n} \eta_{\ell}\right\rfloor$. This works because $\left\lfloor a_{n} \zeta_{\ell}\right\rfloor$ is a subset of $\left\lfloor n \zeta_{\ell}\right\rfloor$ that escapes to infinity. Thus for $\widehat{\omega} \in \widehat{\Omega}_{0}$

$$
\begin{aligned}
B_{+}^{\bar{\zeta}_{\ell}}\left(\widehat{\omega}, x, x+e_{1}\right) & \leq \lim _{n \rightarrow \infty}\left(G_{x, v_{n}}(\omega)-G_{x+e_{1}, v_{n}}(\omega)\right) \\
& \leq \varlimsup_{n \rightarrow \infty}\left(G_{x, v_{n}}(\omega)-G_{x+e_{1}, v_{n}}(\omega)\right) \leq B_{-}^{\underline{\eta}_{\ell}}\left(\widehat{\omega}, x, x+e_{1}\right) .
\end{aligned}
$$

Take $\ell \rightarrow \infty$ and apply (5.5) to arrive at (6.2) as stated. (6.3) follows similarly.

\section{Cocycles from queuing fixed points}

This section proves Theorem 5.2. At the end of the section we address briefly the exactly solvable case. By shifting the variables $\left\{\omega_{x}, B_{ \pm}^{\xi}\left(x, x+e_{i}\right)\right\}$ in Theorem 5.2 if necessary, we can assume without loss of generality that $\mathbb{P}\left\{\omega_{0} \geq 0\right\}=1$. Then the weights $\omega_{x}$ can represent service times in queueing theory. We switch to queuing terminology to enable the reader to relate this section to queueing literature.

Consider an infinite sequence of $\cdot / \mathrm{G} / 1 / \infty /$ FIFO queues in tandem. That is, each queue or service station (these terms are used interchangeably) has a general service time distribution (the law of $\omega_{x}$ under $\mathbb{P}$ ), a single server, unbounded room for customers waiting to be served, and customers obey first-in-first-out discipline. The service stations are indexed by $k \in \mathbb{Z}_{+}$and a bi-infinite sequence of customers is indexed by $n \in \mathbb{Z}$. Customers enter the system at station 0 and move from station to station in order. The server at station $k$ serves one customer at a time. Once the service of customer $n$ is complete at station $k$, customer $n$ moves to the back of the queue at station $k+1$ and customer $n+1$ enters service at station $k$ if they were already waiting in the queue. If the queue at station $k$ is empty after the departure of customer $n$, then server $k$ remains idle until customer $n+1$ arrives. Each customer retains their integer label as they move through the system.

The system has two independent inputs: (i) a stationary, ergodic, arrival process $A^{0}=\left\{A_{n, 0}\right\}_{n \in \mathbb{Z}}$ and (ii) i.i.d. service times $\left\{S_{n, k}\right\}_{n \in \mathbb{Z}, k \in \mathbb{Z}_{+}}$with distribution $S_{0,0} \stackrel{d}{=} \omega_{0}$ under $\mathbb{P}$. $A_{n, 0} \geq 0$ is the time between the arrival of customer $n$ and customer $n+1$ at queue 0 . $S_{n, k} \geq 0$ is the amount of time the service of customer $n$ takes at station $k$. Assume

$$
E\left[S_{0,0}\right]=m_{0}<E\left[A_{0,0}\right]<\infty
$$

The development begins with the waiting times at station 0. Define the stationary, ergodic process $\left\{W_{n, 0}\right\}_{n \in \mathbb{Z}}$ by

$$
W_{n, 0}=\left(\sup _{j \leq n-1} \sum_{i=j}^{n-1}\left(S_{i, 0}-A_{i, 0}\right)\right)^{+}
$$


By the ergodic theorem and (7.1), $W_{n, 0}<\infty$ a.s. $\forall n \in \mathbb{Z} .\left\{W_{n, 0}\right\}$ satisfies Lindley's equation:

$$
W_{n+1,0}=\left(W_{n, 0}+S_{n, 0}-A_{n, 0}\right)^{+}
$$

The interpretation of $W_{n, 0}$ as the waiting time of customer $n$ is natural from this equation. If $W_{n, 0}+S_{n, 0}<A_{n, 0}$ then customer $n$ leaves station 0 before customer $n+1$ arrives, and consequently customer $n+1$ has no wait and $W_{n+1,0}=0$. In the complementary case customer $n+1$ waits time $W_{n+1,0}=W_{n, 0}+S_{n, 0}-A_{n, 0}$ before entering service at station 0 .

Lemma $7.1 n^{-1} W_{n, 0} \rightarrow 0$ almost surely as $n \rightarrow \infty$.

Proof Abbreviate $U_{n}=S_{n, 0}-A_{n, 0}$. For $a \geq 0$ and $\varepsilon>0$ define

$$
W_{0}^{\varepsilon}(a)=a \quad \text { and } \quad W_{n+1}^{\varepsilon}(a)=\left(W_{n}^{\varepsilon}(a)+U_{n}-E\left(U_{0}\right)+\varepsilon\right)^{+} \text {for } n \geq 0 .
$$

Check inductively that

$$
W_{n}^{\varepsilon}(0)=\left(\max _{0 \leq m<n} \sum_{k=m}^{n-1}\left[U_{k}-E\left(U_{0}\right)+\varepsilon\right]\right)^{+} .
$$

Consequently

$$
W_{n}^{\varepsilon}(a) \geq W_{n}^{\varepsilon}(0) \geq \sum_{k=0}^{n-1}\left[U_{k}-E\left(U_{0}\right)+\varepsilon\right] \longrightarrow \infty \quad \text { as } n \rightarrow \infty .
$$

Thus $W_{n}^{\varepsilon}(a)>0$ for large $n$ which implies, from its definition, that for large $n$

$$
W_{n+1}^{\varepsilon}(a)=W_{n}^{\varepsilon}(a)+U_{n}-E\left(U_{0}\right)+\varepsilon .
$$

Another application of the ergodic theorem gives $n^{-1} W_{n}^{\varepsilon}(a) \rightarrow \varepsilon \mathbb{P}$-a.s. as $n \rightarrow \infty$.

Now for the conclusion. Since $W_{0,0}=W_{0}^{\varepsilon}\left(W_{0,0}\right)$, we can check inductively that

$$
\begin{aligned}
W_{n+1,0} & =\left(W_{n, 0}+U_{n}\right)^{+} \leq\left(W_{n}^{\varepsilon}\left(W_{0,0}\right)+U_{n}\right)^{+} \\
& \leq\left(W_{n}^{\varepsilon}\left(W_{0,0}\right)+U_{n}-E\left(U_{0}\right)+\varepsilon\right)^{+}=W_{n+1}^{\varepsilon}\left(W_{0,0}\right) .
\end{aligned}
$$

From this, $0 \leq n^{-1} W_{n, 0} \leq n^{-1} W_{n}^{\varepsilon}\left(W_{0,0}\right) \rightarrow \varepsilon$, and we let $\varepsilon \searrow 0$

The stationary and ergodic process $A^{1}=\left\{A_{n, 1}\right\}_{n \in \mathbb{Z}}$ of inter-departure times from queue 0 (equivalently, inter-arrival times at queue 1 ) is defined by

$$
A_{n, 1}=\left(W_{n, 0}+S_{n, 0}-A_{n, 0}\right)^{-}+S_{n+1,0} .
$$


To see that this definition agrees with common sense, consider the two possible scenarios. If $W_{n+1,0}>0$ from (7.3) then customer $n+1$ is already waiting and goes immediately into service after the departure of customer $n$. The time $A_{n, 1}$ between the departures of customers $n$ and $n+1$ is then exactly the service time $S_{n+1,0}$ of customer $n+1$. In the complementary case $W_{n+1,0}=0$, server 0 is idle for time $A_{n, 0}-S_{n, 0}-W_{n, 0}$ before customer $n+1$ arrives. The time between the departures is this idle time plus the service time $S_{n+1,0}$.

Combining Eqs. (7.3) and (7.4) and iterating gives

$$
W_{1,0}+S_{1,0}+\sum_{i=1}^{n} A_{i, 1}=W_{n+1,0}+S_{n+1,0}+\sum_{i=1}^{n} A_{i, 0} \quad \text { for } n \geq 1 .
$$

This and Lemma 7.1 imply $E\left[A_{0,1}\right]=E\left[A_{0,0}\right]$. (In the queueing literature, this has been observed in [40].)

These steps are repeated successively at each station $k=1,2,3, \ldots$ The stationary, ergodic arrival process $A^{k}=\left\{A_{n, k}\right\}_{n \in \mathbb{Z}}$ at station $k$ is the departure process from station $k-1$. $A_{n, k}$ is the inter-arrival time between customers $n$ and $n+1$ at station $k$, or, equivalently, the inter-departure time between customers $n$ and $n+1$ from station $k-1$. $A^{k}$ is independent of the service times $\left\{S_{n, j}\right\}_{n \in \mathbb{Z}, j \geq k}$ because $A^{k}$ was constructed as a function of the given initial arrival process $A^{0}$ and the service times $\left\{S_{n, j}\right\}_{n \in \mathbb{Z}, 0 \leq j \leq k-1}$. $W_{n, k}$ is the waiting time of customer $n$ at queue $k$, that is, the time between the arrival of customer $n$ at station $k$ and the beginning of their service at station $k$. These are defined by

$$
W_{n, k}=\left(\sup _{j \leq n-1} \sum_{i=j}^{n-1}\left(S_{i, k}-A_{i, k}\right)\right)^{+}, \quad n \in \mathbb{Z}
$$

Properties $W_{n, k}<\infty$, Lemma 7.1, and $E\left[A_{n, k}\right]=E\left[A_{0,0}\right]$ are preserved along the way. The total time customer $n$ spends at station $k$ is the sojourn time $W_{n, k}+S_{n, k}$.

This procedure constructs the process $\left\{A_{n, k}, S_{n, k}, W_{n, k}\right\}_{n \in \mathbb{Z}, k \in \mathbb{Z}_{+}}$that satisfies the following system of equations:

$$
\begin{aligned}
W_{n+1, k}+S_{n+1, k} & =S_{n+1, k}+\left(W_{n, k}+S_{n, k}-A_{n, k}\right)^{+}, \\
A_{n, k+1} & =\left(W_{n, k}+S_{n, k}-A_{n, k}\right)^{-}+S_{n+1, k}, \\
S_{n+1, k} & =\left(S_{n+1, k}+W_{n+1, k}\right) \wedge A_{n, k+1} .
\end{aligned}
$$

The appearance of $S_{n+1, k}$ on both sides of the first line is intentional. The third equation follows from the first two by taking the minimum of either side. Subtracting the second line from the first line in (7.6) gives the "conservation law"

$$
W_{n+1, k}+S_{n+1, k}+A_{n, k}=W_{n, k}+S_{n, k}+A_{n, k+1} .
$$

As a product of an ergodic process and an i.i.d. one, the input $\left\{A_{n, 0}, S_{n, k}\right\}_{n \in \mathbb{Z}, k \in \mathbb{Z}_{+}}$is stationary and ergodic under translations of the $n$-index. Consequently so is the entire 
queuing system. A fixed point is a distribution of $\left\{A_{n, 0}\right\}_{n \in \mathbb{Z}}$ such that the system is also stationary under translations of the $k$-index.

The next four statements summarize the situation with fixed points, quoted from

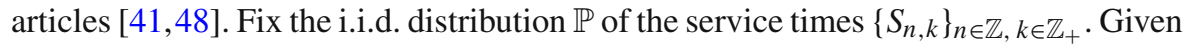
a stationary ergodic probability measure $\mu$ on $\mathbb{R}^{\mathbb{Z}}$, consider the random variables $\left\{A_{n, 0}, S_{n, 0}, W_{n, 0}, A_{n, 1}\right\}_{n \in \mathbb{Z}}$ where $\left\{A_{n, 0}\right\}_{n \in \mathbb{Z}} \sim \mu$ are independent of $\left\{S_{n, 0}\right\}_{n \in \mathbb{Z}} \sim \mathbb{P}$, $W_{n, 0}$ is defined via (7.2), and $A_{n, 1}$ is defined via (7.4). Let $\Phi(\mu)$ denote the distribution of the sequence $\left\{A_{n, 1}\right\}_{n \in \mathbb{Z}}$. $\Phi$ is the queueing operator whose fixed points are the focus now.

Let $\mathscr{M}_{e}^{\alpha}\left(\mathbb{R}^{\mathbb{Z}}\right)$ be the space of translation-ergodic probability measures $\mu$ on $\mathbb{R}^{\mathbb{Z}}$ with marginal mean $E^{\mu}\left[A_{0,0}\right]=\alpha$. From the discussion above we know that $\Phi$ maps $\mathscr{M}_{e}^{\alpha}\left(\mathbb{R}^{\mathbb{Z}}\right)$ into $\mathscr{M}_{e}^{\alpha}\left(\mathbb{R}^{\mathbb{Z}}\right)$. We are interested in ergodic fixed points, so we define

$$
\mathscr{A}=\left\{\alpha \in\left(m_{0}, \infty\right): \exists \mu \in \mathscr{M}_{e}^{\alpha}\left(\mathbb{R}^{\mathbb{Z}}\right) \text { such that } \Phi(\mu)=\mu\right\}
$$

Theorem 7.2 [48, Thm. 1] Let $\alpha \in \mathscr{A}$. Then there exists a unique $\mu^{\alpha} \in \mathscr{M}_{e}^{\alpha}\left(\mathbb{R}^{\mathbb{Z}}\right)$ with $\Phi\left(\mu^{\alpha}\right)=\mu^{\alpha}$. Furthermore, let $A^{0}=\left\{A_{n, 0}\right\}_{n \in \mathbb{Z}}$ be ergodic with mean $E\left[A_{0,0}\right]=$ $\alpha$ and $\left\{S_{n, k}\right\}_{n \in \mathbb{Z}, k \in \mathbb{Z}_{+}}$i.i.d. with distribution $\mathbb{P}$ and independent of $A^{0}$. Let $A^{k}=$ $\left\{A_{n, k}\right\}_{n \in \mathbb{Z}}, k \in \mathbb{N}$, be defined via inductions (7.2) and (7.6). Then as $k \rightarrow \infty$ the distributions of $A^{k}$ converge weakly to $\mu^{\alpha}$.

Theorem 7.3 [41, Thm. 5.1 and 6.4 and Lm. 6.3(a)] Set $\mathscr{A}$ is a nonempty, closed subset of $\left(m_{0}, \infty\right)$, inf $\mathscr{A}=m_{0}$, and $\sup \mathscr{A}=\infty$. If $\alpha<\beta$ are both in $\mathscr{A}$ then $\mu^{\alpha} \leq \mu^{\beta}$ in the usual sense of stochastic ordering.

Lemma 7.4 [41, Lm. 6.3(b)] Let $\alpha \in \mathscr{A}, A^{0} \sim \mu^{\alpha}$, and $\left\{S_{n, k}\right\} \sim \mathbb{P}$ independent of $A^{0}$. Define $W_{n, 0}$ via (7.2). Then

$$
E^{\mu^{\alpha} \otimes \mathbb{P}}\left[W_{0,0}+S_{0,0}\right]=f(\alpha)
$$

Suppose $\alpha \in\left(m_{0}, \infty\right) \cap \mathscr{A}^{c}$. Let

$$
\underline{\alpha}=\sup \left(\mathscr{A} \cap\left(m_{0}, \alpha\right]\right) \in \mathscr{A} \text { and } \bar{\alpha}=\inf (\mathscr{A} \cap[\alpha, \infty)) \in \mathscr{A}
$$

$t=(\bar{\alpha}-\alpha) /(\bar{\alpha}-\underline{\alpha})$ and $\mu^{\alpha}=t \mu^{\underline{\alpha}}+(1-t) \mu^{\bar{\alpha}}$. Now $\mu^{\alpha}$ is a mean $\alpha$ fixed point of $\Phi$. This fixed point is again attractive, in the following sense.

Theorem 7.5 [41, Prop. 6.5] Let $\alpha>m_{0}$. Let $A^{0}=\left\{A_{n, 0}\right\}_{n \in \mathbb{Z}}$ be ergodic with mean $E\left[A_{0,0}\right]=\alpha$ and $\left\{S_{n, k}\right\}_{n \in \mathbb{Z}, k \in \mathbb{Z}_{+}}$i.i.d. with distribution $\mathbb{P}$ and independent of the $\left\{A_{n, 0}\right\}$-process. Let $\left\{A_{n, k}: n \in \mathbb{Z}, k \in \mathbb{N}\right\}$ be defined via inductions (7.2) and (7.6). Then as $k \rightarrow \infty$ the Cesàro mean of the distributions of $\left\{A_{n, k}\right\}_{n \in \mathbb{Z}}$ converges weakly to $\mu^{\alpha}$.

Based on the development in [41] we deduce auxiliary facts.

Lemma 7.6 (a) Let $\underline{\alpha}<\bar{\alpha}$ be points in $\mathscr{A}$ such that $(\underline{\alpha}, \bar{\alpha}) \subset \mathscr{A}^{c}$. Then $f$ is linear on the interval $[\underline{\alpha}, \bar{\alpha}]$. 
(b) Let $\xi \in \mathscr{D}, s=\xi \cdot e_{1} / \xi \cdot e_{2}$ and $\alpha=\gamma^{\prime}(s)$. Then $\alpha \in \mathscr{A}$.

Proof Part (a). Let $0<t<1$ and $\alpha=t \underline{\alpha}+(1-t) \bar{\alpha}$. In the notation of [41], consider a sequence of tandem queues $\left(A^{k}, S^{k}, W^{k}, A^{k+1}\right)_{k \in \mathbb{Z}_{+}}$where the initial arrival process $A^{0}=\left\{A_{n, 0}\right\}_{n \in \mathbb{Z}}$ is ergodic with mean $E\left(A_{n, 0}\right)=\alpha$, the service times $\left\{S^{k}\right\}_{k \in \mathbb{Z}_{+}}=\left\{S_{n, k}\right\}_{n \in \mathbb{Z}, k \in \mathbb{Z}_{+}}$are independent of $A^{0}$ and i.i.d. $\mathbb{P}$-distributed, and the remaining variables are defined iteratively. Let $(\widehat{A}, \widehat{S}, \widetilde{W}, \widetilde{D})$ denote a weak limit point of the Cesàro averages of the distributions of $\left(A^{k}, S^{k}, W^{k}, A^{k+1}\right)$. (The tightness argument is on p. 2225 of [41].) Then, as shown in [41, eqn. (29)] in the course of the proof of their Theorem 5.1, $\widetilde{W}=\Psi(\widehat{A}, \widehat{S})$ where the mapping $\Psi$ encodes definition (7.2). By Theorem 7.5 [41, Prop. 6.5] the distribution of $\widehat{A}$ is $t \mu^{\underline{\alpha}}+(1-t) \mu^{\bar{\alpha}}$. By [41, Theorem 4.1],

$$
n^{-1} \sum_{k=0}^{n-1} W_{0, k} \rightarrow M(\alpha) \equiv f(\alpha)-m_{0} \quad \text { almost surely. }
$$

Combine these facts as follows. First

$$
\begin{aligned}
E\left(\widetilde{W}_{0}\right) & =E\left[\Psi(\widehat{A}, \widehat{S})_{0}\right]=t E^{\mu^{\underline{\alpha}} \otimes \mathbb{P}}\left[\Psi(\widehat{A}, \widehat{S})_{0}\right]+(1-t) E^{\mu^{\bar{\alpha}} \otimes \mathbb{P}}\left[\Psi(\widehat{A}, \widehat{S})_{0}\right] \\
& =t M(\underline{\alpha})+(1-t) M(\bar{\alpha})
\end{aligned}
$$

where the last equality comes from [41, Lemma 6.3(b)] restated as Lemma 7.4 above. The weak limit, combined with the law of large numbers (7.9) and dominated convergence, gives, for any $c<\infty$ and along a subsequence,

$$
\begin{aligned}
E\left(\widetilde{W}_{0} \wedge c\right) & =\lim _{n \rightarrow \infty} n^{-1} \sum_{k=0}^{n-1} E\left(W_{0, k} \wedge c\right) \leq \lim _{n \rightarrow \infty} E\left[\left(n^{-1} \sum_{k=0}^{n-1} W_{0, k}\right) \wedge c\right]=M(\alpha) \wedge c \\
& \leq M(\alpha) .
\end{aligned}
$$

Letting $c \nearrow \infty$ gives

$$
t M(\underline{\alpha})+(1-t) M(\bar{\alpha}) \leq M(\alpha) .
$$

Since $M$ is convex and $f$ differs from $M$ by a constant, this implies $f(\alpha)=t f(\underline{\alpha})+$ $(1-t) f(\bar{\alpha})$ and completes the proof of part (a).

Part (b). If $\alpha \in \mathscr{A}^{c}$, there exist $\underline{\alpha}<\bar{\alpha}$ in $\mathscr{A}$ such that $\alpha \in(\underline{\alpha}, \bar{\alpha}) \subset \mathscr{A}^{c}$. By part (a) $f$ is linear on $[\underline{\alpha}, \bar{\alpha}]$. Basic convex analysis implies that $\gamma$ has multiple tangent slopes at $s$ and hence cannot be differentiable at $s$.

Given $\alpha>m_{0}$, let $\left\{A_{n, 0}\right\}_{n \in \mathbb{Z}} \sim \mu^{\alpha}$ and i.i.d. $\left\{S_{n, k}\right\}_{n \in \mathbb{Z}, k \in \mathbb{Z}_{+}} \sim \mathbb{P}$ be independent. Define $\left\{W_{n, k}, A_{n, k+1}\right\}_{n \in \mathbb{Z}, k \in \mathbb{Z}_{+}}$using (7.5) and (7.6). Because $\Phi\left(\mu^{\alpha}\right)=\mu^{\alpha}$, $\left\{A_{n, k}, S_{n, k}, W_{n, k}\right\}_{n \in \mathbb{Z}, k \in \mathbb{Z}_{+}}$is stationary in $n$ and $k$. Extend this to the stationary process 
$(A, S, W)=\left\{A_{n, k}, S_{n, k}, W_{n, k}\right\}_{n, k \in \mathbb{Z}}$ indexed by $\mathbb{Z}^{2}$. Define another $\mathbb{Z}^{2}$-indexed stationary process $(\tilde{A}, \widetilde{S}, \widetilde{W})$ by

$$
\left(\widetilde{A}_{i, j}, \widetilde{S}_{i, j}, \widetilde{W}_{i, j}\right)=\left(W_{j-1, i+1}+S_{j-1, i+1}, S_{j, i}, A_{j-1, i+1}-S_{j, i}\right), \quad(i, j) \in \mathbb{Z}^{2} .
$$

Lemma 7.7 Suppose $\alpha \in \mathscr{A}$. Then the process $(A, S, W)$ is ergodic under translation $T_{e_{1}}$, and also ergodic under $T_{e_{2}}$. Furthermore, $f(\alpha) \in \mathscr{A} .(\widetilde{A}, \widetilde{S}, \widetilde{W})$ is a stationary queueing system where $\left\{\widetilde{A}_{n, 0}\right\}_{n \in \mathbb{Z}}$ has distribution $\mu^{f(\alpha)}$, and is also ergodic under both $T_{e_{1}}$ and $T_{e_{2}}$.

Proof The queueing construction gives $T_{e_{1}}$-ergodicity of $\left\{A_{n, k}, S_{n, k}, W_{n, k}\right\}_{n \in \mathbb{Z}, k \geq \ell}$ for any $\ell \in \mathbb{Z}$. Let $B$ be a $T_{e_{1}}$-invariant event of the full process $\left\{A_{n, k}, S_{n, k}, W_{n, k}\right\}_{n, k \in \mathbb{Z}}$. Write $\mathscr{G}_{\ell}$ for the $\sigma$-algebra generated by $\left\{A_{n, k}, S_{n, k}, W_{n, k}\right\}_{n \in \mathbb{Z}, k \geq \ell}$. The conditional expectations $E\left(\mathbb{1}_{B} \mid \mathscr{G}_{\ell}\right)$ are $T_{e_{1}}$-invariant, hence a.s. constant by the ergodicity proved thus far. $E\left(\mathbb{1}_{B} \mid \mathscr{G}_{\ell}\right) \rightarrow \mathbb{1}_{B}$ almost surely as $\ell \rightarrow-\infty$, and consequently $\mathbb{1}_{B}$ is a.s. constant. This completes the proof of ergodicity under $T_{e_{1}}$.

To get ergodicity under $T_{e_{2}}$ we transpose, and that leads us to look at $(\widetilde{A}, \widetilde{S}, \widetilde{W})$ of (7.10). To see that $(\widetilde{A}, \widetilde{S}, \widetilde{W})$ is another queueing system with the same i.i.d. service time distribution $\widetilde{S}_{i, j}=S_{j, i}$, we need to check three items.

(i) Independence of $\left\{\widetilde{A}_{i, \ell}\right\}_{i \in \mathbb{Z}}$ and $\left\{\widetilde{S}_{i, j}\right\}_{i \in \mathbb{Z}, j \geq \ell}$, for each $\ell \in \mathbb{Z}$. This follows from the structure of equations (7.6) and the independence of the $\left\{S_{i, j}\right\}$.

(ii) $\widetilde{A}_{i, j+1}=\left(\widetilde{W}_{i j}+\widetilde{S}_{i j}-\widetilde{A}_{i j}\right)^{-}+\widetilde{S}_{i+1, j}$. This follows from the top equation of (7.6).

(iii) The third point needed is

$$
\widetilde{W}_{k+1, j}=\left(\sup _{n: n \leq k} \sum_{i=n}^{k}\left(\widetilde{S}_{i j}-\widetilde{A}_{i j}\right)\right)^{+}
$$

This needs a short argument. Fix $k, j$. The middle equation of (7.6) gives

$$
\widetilde{W}_{i j}=\left(\widetilde{W}_{i-1, j}+\widetilde{S}_{i-1, j}-\widetilde{A}_{i-1, j}\right)^{+}
$$

which can be iterated to give

$$
\widetilde{W}_{k+1, j}=\left(\left\{\widetilde{W}_{\ell j}+\sum_{i=\ell}^{k}\left(\widetilde{S}_{i j}-\widetilde{A}_{i j}\right)\right\} \vee\left\{\max _{n: \ell<n \leq k} \sum_{i=n}^{k}\left(\widetilde{S}_{i j}-\widetilde{A}_{i j}\right)\right\}\right)^{+} \quad \text { for } \ell \leq k .
$$

Thus (7.11) follows if $\widetilde{W}_{\ell j}=0$ for some $\ell \leq k$. Suppose on the contrary that $\widetilde{W}_{i j}>0$ for all $i \leq k$. Apply (7.12) to all $\widetilde{W}_{i j}$ for $n<i \leq k$ and divide by $|n|$ to get

$$
\frac{\widetilde{W}_{k j}}{|n|}=\frac{\widetilde{W}_{n j}}{|n|}+\frac{1}{|n|} \sum_{i=n}^{k-1}\left(\widetilde{S}_{i j}-\widetilde{A}_{i j}\right)
$$


which is the same as

$$
\frac{A_{j-1, k+1}}{|n|}-\frac{S_{j k}}{|n|}=\frac{A_{j-1, n+1}}{|n|}-\frac{S_{j n}}{|n|}+\frac{1}{|n|} \sum_{i=n}^{k-1}\left(S_{j i}-W_{j-1, i+1}-S_{j-1, i+1}\right) .
$$

Let $n \rightarrow-\infty$. The i.i.d. property of the $\left\{S_{i j}\right\}$ and Theorem 4.1 of [41] quoted as (7.9) above, combined with (7.8) from above, give the limit in probability

$$
\lim _{n \rightarrow-\infty} \frac{1}{|n|} \sum_{i=n}^{k-1} \widetilde{A}_{i j}=\lim _{n \rightarrow-\infty} \frac{1}{|n|} \sum_{i=n}^{k-1}\left(W_{j-1, i+1}+S_{j-1, i+1}\right)=f(\alpha) .
$$

The four leftmost terms of (7.13) vanish as $n \rightarrow-\infty$ (by stationarity and finite expectations). Hence letting $n \rightarrow-\infty$ in (7.13) along a suitable subsequence leads to $0=m_{0}-f(\alpha)<0$ (the last inequality from Lemma 4.3). This contradiction verifies (7.11).

At this point we have shown that the stationary process $\left\{\widetilde{A}_{n, 0}\right\}_{n \in \mathbb{Z}}$ is a fixed point for $\Phi$ with the deterministic pathwise limit (7.14). By Prop. 4.4 of [41] the process $\left\{\widetilde{A}_{n, 0}\right\}_{n \in \mathbb{Z}}$ must be ergodic. We have shown that $f(\alpha) \in \mathscr{A}$. The part of the lemma already proved gives the ergodicity of the process

$$
\left\{\widetilde{A}_{i j}, \widetilde{S}_{i j}, \widetilde{W}_{i j}\right\}=\left\{W_{j-1, i+1}+S_{j-1, i+1}, S_{j, i}, A_{j-1, i+1}-S_{j, i}\right\}
$$

under translations of the index $i$. Since ergodicity is preserved by mappings that respect translations, a suitable mapping of the right-hand side above gives the $T_{e_{2}}$-ergodicity of $\left\{A_{n k}, S_{n k}, W_{n k}\right\}$.

Proof of Theorem 5.2 We begin by constructing a convenient countable subset $\mathscr{A}_{0}$ of $\mathscr{A}$. Let $\mathscr{U}_{0}$ be a countable dense subset of ri $\mathscr{U}$ such that $\mathscr{U}_{0}$ contains

(i) all (at most countably many) points of nondifferentiability of $g_{\mathrm{pp}}$,

(ii) all endpoints of nondegenerate intervals on which $g_{\mathrm{pp}}$ is linear (recall (4.4)), and

(iii) a countable dense subset of points of differentiability of $g_{\mathrm{pp}}$.

Then put

$$
\mathscr{A}_{0}=\left\{\gamma^{\prime}(s \pm):\left(\frac{s}{1+s}, \frac{1}{1+s}\right) \in \mathscr{U}_{0}\right\} .
$$

$\mathscr{A}_{0} \subset \mathscr{A}$ by virtue of Lemma 7.6(b) and the closedness of $\mathscr{A}$ in $\left(m_{0}, \infty\right)$.

We construct a coupled process

$$
\left\{\left(A^{\alpha}, S, W^{\alpha}\right): \alpha \in \mathscr{A}_{0}\right\}=\left\{A_{n, k}^{\alpha}, S_{n, k}, W_{n, k}^{\alpha}:(n, k) \in \mathbb{Z}^{2}, \alpha \in \mathscr{A}_{0}\right\}
$$

whose distribution $\bar{P}$ on $\mathbb{R}^{\mathscr{A}_{0} \times \mathbb{Z}^{2}} \times \mathbb{R}^{\mathbb{Z}^{2}} \times \mathbb{R}^{\mathscr{A}_{0} \times \mathbb{Z}^{2}}$ is invariant under translations of the $(n, k)$ index over $\mathbb{Z}^{2}$ and that has the following properties. For each $(n, k) \in \mathbb{Z}^{2}$ we have the inequalities

$$
A_{n, k}^{\alpha} \leq A_{n, k}^{\beta} \quad \text { and } \quad W_{n, k}^{\alpha} \geq W_{n, k}^{\beta} \quad \text { for } \alpha<\beta .
$$


For each $\alpha \in \mathscr{A}_{0}$ the marginal process $\left(A^{\alpha}, S, W^{\alpha}\right)$ is a stationary queueing process of the type described in Lemma 7.7, stationary and ergodic under both translations, with $\left\{A_{n, 0}^{\alpha}\right\}_{n \in \mathbb{Z}} \sim \mu^{\alpha}$ and $\left\{S_{0, k}+W_{0, k}^{\alpha}\right\}_{k \in \mathbb{Z}} \sim \mu^{f(\alpha)} . \bar{P}$ comes from a subsequential Cesàro limit of queueing processes followed by Kolmogorov extension to the full lattice.

Start by taking for each $\alpha \in \mathscr{A}_{0}$ the constant initial inter-arrival process $A_{n, 0}^{\alpha}=\alpha$. Use the iterative equations to construct $\left(A^{\alpha, k}, S^{k}, W^{\alpha, k}\right)=\left\{A_{n, k}^{\alpha}, S_{n, k}, W_{n, k}^{\alpha}\right\}_{n \in \mathbb{Z}}$ for $k \in \mathbb{Z}_{+}$. Use the same version of the service times $\left\{S_{n, k}\right\}$ for each $\alpha \in \mathscr{A}_{0}$. Inequalities (7.16) are true for the $A$-processes at $k=0$ by construction. They are propagated for all $k \in \mathbb{Z}_{+}$by Eqs. (7.5) and (7.6).

Let $Q^{\ell}$ be the joint distribution of $\left\{\left(A^{\alpha, \ell+k}, S^{\ell+k}, W^{\alpha, \ell+k}\right): \alpha \in \mathscr{A}_{0}, k \geq 0\right\}$, a probability measure on the countable product space $\mathbb{R}^{\mathscr{A}_{0} \times \mathbb{Z} \times \mathbb{Z}_{+}} \times \mathbb{R}^{\mathbb{Z} \times \overline{\mathbb{Z}}_{+} \times}$

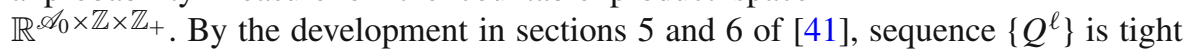
and for each $\alpha \in \mathscr{A}_{0}, A^{\alpha, k}$ converges weakly to $\mu^{\alpha}$ as $k \rightarrow \infty$ and the process $\left(A^{\alpha, \ell+k}, S^{\ell+k}, W^{\alpha, \ell+k}\right)_{k \geq 0}$ converges weakly to a stationary queueing process as $\ell \rightarrow \infty$. In order to get a limit where the joint distribution between different $\alpha$ values is also invariant under shifts of the $k$-index, we perform one averaging: let $\bar{Q}^{n}=n^{-1} \sum_{\ell=0}^{n-1} Q^{\ell}$. The effect of shifting the $k$-index is $\bar{Q}^{n} \circ T_{e_{2}}^{-1}=n^{-1} \sum_{\ell=1}^{n} Q^{\ell}$. Thus any limit point $\bar{Q}$ of $\left\{\bar{Q}^{n}\right\}$ is invariant under shifts of the $k$-index. This invariance extends $\bar{Q}$ to negative $k$-values and gives an invariant measure $\bar{P}$ indexed by $(n, k) \in \mathbb{Z}^{2}$. The almost sure inequalities (7.16) are preserved in this construction. $\bar{P}$ has the properties described below (7.16).

Define the following mapping from the coordinates $\left\{\left(A^{\alpha}, S, W^{\alpha}\right): \alpha \in \mathscr{A}_{0}\right\}$ to the

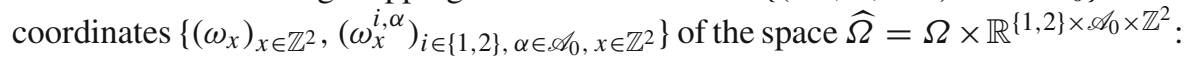
for $(n, k) \in \mathbb{Z}^{2}$ and $\alpha \in \mathscr{A}_{0}$,

$$
\left(\omega_{n, k}, \omega_{n, k}^{1, \alpha}, \omega_{n, k}^{2, \alpha}\right)=\left(S_{-n,-k}, A_{-n-1,-k+1}^{\alpha}, W_{-n,-k}^{\alpha}+S_{-n,-k}\right) .
$$

Let $\widehat{\mathbb{P}}$ be the distribution induced on $\widehat{\Omega}$ by this mapping, from the joint distribution $\bar{P}$ of the coupled stationary queueing processes.

The probability space $(\widehat{\Omega}, \widehat{\mathcal{S}}, \widehat{\mathbb{P}})$ of Theorem 5.2 has now been constructed. For $\xi \in \mathscr{U}_{0}$ and $i=1,2$ define the functions $B_{ \pm}^{\xi}\left(\widehat{\omega}, x, x+e_{i}\right)$ as the following coordinate projections:

$$
B_{ \pm}^{\xi}\left(\widehat{\omega}, x, x+e_{i}\right)=\omega_{x}^{i, \gamma^{\prime}(s \pm)} \quad \text { for } s=\xi \cdot e_{1} / \xi \cdot e_{2}
$$

The set $\mathscr{A}_{0}$ was constructed to ensure $\gamma^{\prime}(s \pm) \in \mathscr{A}_{0}$ for each $\xi \in \mathscr{U}_{0}$ so these functions are well-defined. For each $\xi \in \mathscr{U}_{0}$ and $x, y \in \mathbb{Z}^{d}$, follow the same deterministic procedure to extend these functions to $B_{ \pm}^{\xi}(\widehat{\omega}, x, y)$ for all $x, y \in \mathbb{Z}^{d}$. For example, through these steps:

- Set $B_{ \pm}^{\xi}(\widehat{\omega}, x, x)=0$. For $x \leq y$ fix a path $x=x_{0}, x_{1}, \ldots, x_{m}=y$ such that all $e_{1}$ steps come before $e_{2}$ steps and set $B_{ \pm}^{\xi}(\widehat{\omega}, x, y)=\sum_{i=0}^{m-1} B_{ \pm}^{\xi}\left(\widehat{\omega}, x_{i}, x_{i+1}\right)$. If $y \leq x$, set $B_{ \pm}^{\xi}(\widehat{\omega}, x, y)=-B_{ \pm}^{\xi}(\widehat{\omega}, y, x)$. 
- If there is no path between $x$ and $y$, let $z=\left(x \cdot e_{1} \wedge y \cdot e_{1}, x \cdot e_{2} \wedge y \cdot e_{2}\right)$ and set $B_{ \pm}^{\xi}(\widehat{\omega}, x, y)=B_{ \pm}^{\xi}(\widehat{\omega}, x, z)+B_{ \pm}^{\xi}(\widehat{\omega}, z, y)$.

The remainder of the proof of Theorem 5.2 consists of two steps: (a) verification that the processes $B_{ \pm}^{\xi}(x, y)$ thus defined for $\xi \in \mathscr{U}_{0}$ satisfy all the properties required by Theorem 5.2 and (b) definition of $B_{ \pm}^{\xi}(x, y)$ for all $\xi \in$ ri $\mathscr{U}$ through right limits followed by another verification of the required properties.

In part (i) of Theorem 5.2 the stationarity and ergodicity of each process

$$
\psi_{x}^{ \pm, \xi}(\widehat{\omega})=\left(\omega_{x}, B_{ \pm}^{\xi}\left(x, x+e_{1}\right), B_{ \pm}^{\xi}\left(x, x+e_{2}\right)\right)
$$

under both translations $T_{e_{1}}$ and $T_{e_{2}}$ are a consequence of Lemma 7.7. The independence claim follows from the queuing construction. For any given $(n, k)$ and $m<-k$, $A_{-n-1,-k}^{\alpha}$ is a function of $\left(\left\{A_{i, m}^{\alpha}\right\}_{i \leq-n-1},\left\{S_{i, j}\right\}_{i \leq-n, m \leq j \leq-k-1}\right)$ and $W_{-n-1,-k}^{\alpha}$ is a function of $\left(\left\{A_{i, m}^{\alpha}\right\}_{i \leq-n-2},\left\{S_{i, j}\right\}_{i \leq-n-1, m \leq j \leq-k}\right)$. These inputs are independent of $S_{-n,-k}$.

Part (ii) of Theorem 5.2 requires the cocycle properties. For a given $\xi \in \mathscr{U}_{0}$, conservation law (7.7) translates into the $\widehat{\mathbb{P}}$-almost sure property

$$
B_{ \pm}^{\xi}\left(x, x+e_{2}\right)+B_{ \pm}^{\xi}\left(x+e_{2}, x+e_{1}+e_{2}\right)=B_{ \pm}^{\xi}\left(x, x+e_{1}\right)+B_{ \pm}^{\xi}\left(x+e_{1}, x+e_{1}+e_{2}\right)
$$

Thus $\left\{B_{ \pm}^{\xi}(x, y)\right\}_{x, y \in \mathbb{Z}^{2}}$ is additive. Stationarity came in the previous paragraph and integrability is the integrability of the $A, S$ and $W$ variables.

The mean vectors satisfy

$$
\begin{aligned}
h_{ \pm}(\xi) & =-\left(\widehat{\mathbb{E}}\left[B_{ \pm}^{\xi}\left(0, e_{1}\right)\right], \widehat{\mathbb{E}}\left[B_{ \pm}^{\xi}\left(0, e_{2}\right)\right]\right)=-\left(E\left[A_{0,0}^{\gamma^{\prime}(s \pm)}\right], E\left[W_{0,0}^{\gamma^{\prime}(s \pm)}+S_{0,0}\right]\right) \\
& =-\left(\gamma^{\prime}(s \pm), f\left(\gamma^{\prime}(s \pm)\right)\right)=-\nabla g_{\mathrm{pp}}(\xi \pm) .
\end{aligned}
$$

The fact that one-sided gradients satisfy the duality (2.7) is basic convex analysis.

The bottom equation of (7.6) translates into the potential-recovery property

$$
\omega_{x}=B_{ \pm}^{\xi}\left(x, x+e_{1}\right) \wedge B_{ \pm}^{\xi}\left(x, x+e_{2}\right) \quad \widehat{\mathbb{P}}-\text { a.s. }
$$

Part (ii) of Theorem 5.2 has been verified for $B_{ \pm}^{\xi}(\widehat{\omega}, x, y)$ for $\xi \in \mathscr{U}_{0}$.

Part (iii) of Theorem 5.2 is the equality of cocycles that share the mean vector. This is clear from (7.18) and the construction because $h_{ \pm}(\xi)$ determines $\gamma^{\prime}(s \pm)$.

For the inequalities of part (iv), let $s=\xi \cdot e_{1} / \xi \cdot e_{2}$ and $t=\zeta \cdot e_{1} / \zeta \cdot e_{2}$ for $\xi, \zeta \in \mathscr{U}_{0}$. Then $\xi \cdot e_{1}<\zeta \cdot e_{1}$ implies $s<t$. By concavity $\gamma^{\prime}(s-) \geq \gamma^{\prime}(s+) \geq \gamma^{\prime}(t-)$ and the first inequality of (7.16) gives $A_{n, k}^{\gamma^{\prime}(s-)} \geq A_{n, k}^{\gamma^{\prime}(s+)} \geq A_{n, k}^{\gamma^{\prime}(t-)}$ which translates into the first inequality of (5.4). Similarly the second inequality of (7.16) gives the second inequality of (5.4). Let $\widehat{\Omega}_{1}$ be the event on which inequalities (5.4) hold for all countably many $\xi, \zeta \in \mathscr{U}_{0}$. 
For $\zeta \in \mathscr{U}_{0}$ define $Y\left(\widehat{\omega}, \zeta, x, x+e_{1}\right)=\sup _{\xi \in \mathscr{U}_{0}: \xi \cdot e_{1}>\zeta \cdot e_{1}} B_{ \pm}^{\xi}\left(x, x+e_{1}\right)$. Then for any sequence $\xi_{n} \in \mathscr{U}_{0}$ such that $\xi_{n} \cdot e_{1} \searrow \zeta \cdot e_{1}$, by monotonicity

$$
\lim _{n \rightarrow \infty} B_{ \pm}^{\xi_{n}}\left(x, x+e_{1}\right)=Y\left(\widehat{\omega}, \zeta, x, x+e_{1}\right) \leq B_{+}^{\zeta}\left(x, x+e_{1}\right) \text { for all } \widehat{\omega} \in \widehat{\Omega}_{1} .
$$

Monotonicity of the family of cocycles gives a bound that justifies dominated convergence, and hence

$$
\widehat{\mathbb{E}}\left[\lim _{n \rightarrow \infty} B_{ \pm}^{\xi_{n}}\left(x, x+e_{1}\right)\right]=\lim _{n \rightarrow \infty} \gamma^{\prime}\left(s_{n} \pm\right)=\gamma^{\prime}(t+)=\widehat{\mathbb{E}}\left[B_{+}^{\zeta}\left(x, x+e_{1}\right)\right]
$$

Equality of expectations forces a.s. equality in (7.19). We now have a.s. right continuity (5.5) for the case $(x, y)=\left(x, x+e_{1}\right)$. Analogously deduce a.s. right continuity (5.5) for $(x, y)=\left(x, x+e_{2}\right)$, and a.s. left continuity (5.6) for $(x, y)=\left(x, x+e_{i}\right)$. Then a.s. left and right continuity follow for all $(x, y)$ by the construction of $B_{ \pm}^{\xi}(x, y)$ in terms of the nearest-neighbor values $B_{ \pm}^{\xi}\left(x, x+e_{i}\right)$.

Let $\widehat{\Omega}_{0}$ be the full $\widehat{\mathbb{P}}$-measure subset of $\widehat{\Omega}_{1}$ on which limits (5.5) and (5.6) hold for all $\zeta \in \mathscr{U}_{0}$ when $\xi_{n} \rightarrow \zeta$ in $\mathscr{U}_{0}$.

Theorem 5.2 has now been verified for $B_{ \pm}^{\xi}$ for $\xi \in \mathscr{U}_{0}$. The next step defines $B_{ \pm}^{\zeta}$ for $\zeta \in($ ri $\mathscr{U}) \backslash \mathscr{U}$. Since all points of nondifferentiability of $g_{\text {pp }}$ were included in $\mathscr{U}_{0}, \zeta \in \mathscr{D}$. Then we define $B_{ \pm}^{\zeta}$ as equal and denote it by $B^{\zeta}$. We choose right limits for the definition. So for $\zeta \in($ ri $\mathscr{U}) \backslash \mathscr{U}_{0}$ set

$$
\begin{aligned}
B^{\zeta}\left(\widehat{\omega}, x, x+e_{1}\right) & =B_{ \pm}^{\zeta}\left(\widehat{\omega}, x, x+e_{1}\right)=\sup _{\xi \in \mathscr{U}_{0}: \xi \cdot e_{1}>\zeta \cdot e_{1}} B_{ \pm}^{\xi}\left(\widehat{\omega}, x, x+e_{1}\right) \\
B^{\zeta}\left(\widehat{\omega}, x, x+e_{2}\right) & =B_{ \pm}^{\zeta}\left(\widehat{\omega}, x, x+e_{2}\right)=\inf _{\xi \in \mathscr{U}_{0}: \xi \cdot e_{1}>\zeta \cdot e_{1}} B_{ \pm}^{\xi}\left(\widehat{\omega}, x, x+e_{2}\right) .
\end{aligned}
$$

On the event $\widehat{\Omega}_{0}$ of full $\widehat{\mathbb{P}}$-probability defined above, definition (7.21) extends inequalities (5.4) and right limits (5.5) to all $\xi, \zeta \in$ ri $\mathscr{U}$, nearest-neighbor edges $\left(x, x+e_{i}\right)$ and sequences $\xi_{n} \cdot e_{1} \searrow \zeta \cdot e_{1}$. Extend the nearest-neighbor values $B^{\zeta}(x, x+$ $\left.e_{i}\right)$ to all $B^{\zeta}(x, y)$ by the procedure used earlier after (7.18). Then right limits (5.5) work for all $B^{\zeta}(x, y)$ and sequences $\xi_{n} \cdot e_{1} \searrow \zeta \cdot e_{1}$.

Fix $\zeta \in($ ri $\mathscr{U}) \backslash \mathscr{U}_{0}$. We argue that outside a single $\widehat{\mathbb{P}}$-null set specific to $\zeta$, we get the left limit (5.6). Define

$$
\begin{aligned}
& Y\left(\widehat{\omega}, \zeta, x, x+e_{1}\right)=\inf _{\xi \in \mathscr{U}_{0}: \xi \cdot e_{1}<\zeta \cdot e_{1}} B_{ \pm}^{\xi}\left(\widehat{\omega}, x, x+e_{1}\right) \\
& Y\left(\widehat{\omega}, \zeta, x, x+e_{2}\right)=\sup _{\xi \in \mathscr{U}_{0}: \xi \cdot e_{1}<\zeta \cdot e_{1}} B_{ \pm}^{\xi}\left(\widehat{\omega}, x, x+e_{2}\right) .
\end{aligned}
$$

The left limit $Y\left(\widehat{\omega}, \zeta, x, x+e_{i}\right)=\lim _{n \rightarrow \infty} B_{ \pm}^{\zeta_{n}}\left(\widehat{\omega}, x, x+e_{i}\right)$ happens for $\widehat{\omega} \in \widehat{\Omega}_{0}$ by monotonicity, for any sequence $\zeta_{n} \cdot e_{1} \nearrow \zeta \cdot e_{1}$ in ri $\mathscr{U}$. Now set

$$
\widehat{\Omega}^{(\zeta)}=\left\{\widehat{\omega} \in \widehat{\Omega}_{0}: Y\left(\widehat{\omega}, \zeta, x, x+e_{i}\right)=B^{\zeta}\left(\widehat{\omega}, x, x+e_{i}\right) \forall x \in \mathbb{Z}^{2}, i=1,2\right\} .
$$


The monotonicity argument with coinciding expectations used above in (7.20) implies that $\widehat{\mathbb{P}}\left(\widehat{\Omega}^{(\zeta)}\right)=1$. From nearest-neighbor values the limits extend to all $(x, y)$ by the construction, and so on the full measure event $\widehat{\Omega}^{(\zeta)}$ we have (5.6).

We turn to verifying the remaining claims of Theorem 5.2 for the fully defined processes $B_{ \pm}^{\xi}(x, y)$. First, right-continuity in $\xi$ is enough to make $B_{+}^{\xi}(\widehat{\omega}, x, y)$ a jointly Borel function of $(\widehat{\omega}, \xi, x, y)$. Since $B_{-}^{\xi}$ replaces the value of $B_{+}^{\xi}$ with a different Borel function of $(\widehat{\omega}, x, y)$ only at the countably many $\xi \in($ ri $\mathscr{U}) \backslash \mathscr{D}, B_{-}^{\xi}(\widehat{\omega}, x, y)$ is also jointly Borel.

Part (i). Stationarity and the independence claim are preserved by almost sure limits but ergodicity is not. To verify the ergodicity of $\psi_{x}^{\zeta}(\widehat{\omega})=\left(\omega_{x}, B^{\zeta}(\widehat{\omega}, x, x+\right.$ $\left.\left.e_{1}\right), B^{\zeta}\left(\widehat{\omega}, x, x+e_{2}\right)\right)$ under both translations $T_{e_{1}}$ and $T_{e_{2}}$ we return to the queuing picture. The limit (7.21) can also be taken in the queueing processes. First $\mathscr{A}_{0} \ni \alpha_{n}=$ $\gamma^{\prime}\left(s_{n}-\right) \nearrow \gamma^{\prime}(t)=\beta$. Since $\mathscr{A}$ is closed, $\beta \in \mathscr{A}$. Hence there is a stationary queueing process $\left(A^{\beta}, S, W^{\beta}\right)$ that satisfies Lemma 7.7 and that we can include in the coupling with the queueing processes indexed by $\mathscr{A}_{0}$. The coordinatewise monotone a.s. limit $\lim _{n \rightarrow \infty}\left(A^{\alpha_{n}}, S, W^{\alpha_{n}}\right)$ must coincide with $\left(A^{\beta}, S, W^{\beta}\right)$ by the same reasoning used above: there are inequalities, namely $\lim _{n \rightarrow \infty} A_{m, k}^{\alpha_{n}} \leq A_{m, k}^{\beta}$ and $\lim _{n \rightarrow \infty} W_{m, k}^{\alpha_{n}} \geq$ $W_{m, k}^{\beta}$, but the expectations agree and hence force a.s. agreement. The continuous mapping (7.17) transports the distribution of $\left\{\left(S_{-n,-k}, A_{-n-1,-k+1}^{\beta}, W_{-n,-k}^{\beta}+S_{-n,-k}\right)\right.$ : $n, k \in \mathbb{Z}\}$ to the process $\left\{\left(\omega_{x}, B^{\zeta}\left(x, x+e_{1}\right), B^{\zeta}\left(x, x+e_{2}\right)\right): x \in \mathbb{Z}^{2}\right\}$, which thereby inherits from Lemma 7.7 the ergodicity claimed in part (i) of Theorem 5.2.

The cocycle properties and expectations in part (ii) are preserved by the limits. The identities of part (iii) continue to hold without null sets because if vector $h(\zeta)$ is not unique to $B^{\zeta}$, then $\zeta$ lies in the interior of some linear segment ] $\xi^{\prime}$, $\xi^{\prime \prime}$ [ of $g_{\text {pp }}$ with $\xi^{\prime}, \xi^{\prime \prime} \in \mathscr{U}_{0}$ and $\xi^{\prime} \cdot e_{1}<\xi^{\prime \prime} \cdot e_{1}$. The construction $((7.18)$ and (7.21)) then implies that $B^{\zeta}=B_{-}^{\xi^{\prime \prime}}=B_{+}^{\xi^{\prime}}$ for all $\left.\zeta \in\right] \xi^{\prime}, \xi^{\prime \prime}$. The inequalities and limits of parts (iv)-(v) were discussed above. This completes the proof of Theorem 5.2.

\subsection{Exactly solvable models}

We describe briefly how the calculations work in the exactly solvable geometric case discussed in Sect. 3.1. (The exponential case is completely analogous.) The weights $\left\{\omega_{x}\right\}$ are i.i.d. with $\mathbb{P}\left(\omega_{x}=k\right)=\left(1-m_{0}{ }^{-1}\right)^{k-1} m_{0}{ }^{-1}$ for $k \in \mathbb{N}$, mean $m_{0}=\mathbb{E}\left(\omega_{0}\right)>$ 1 and variance $\sigma^{2}=m_{0}\left(m_{0}-1\right)$.

With i.i.d. geometric service times $\left\{S_{n, 0}\right\}$ with mean $m_{0}$, let the initial arrival process $\left\{A_{n, 0}\right\}$ be i.i.d. geometric with mean $\alpha$. Let $J_{n}=S_{n, 0}+W_{n, 0}$. Then equations (7.3) and (7.4) show that the process $\left\{\left(A_{n, 1}, J_{n+1}\right)\right\}_{s n \in \mathbb{Z}}$ is an irreducible aperiodic Markov chain with transition probability

$$
\begin{aligned}
P\left(A_{n, 1}\right. & \left.=b, J_{n+1}=j \mid A_{n-1,1}=a, J_{n}=i\right) \\
& =P\left\{\left(A_{n, 0}-i\right)^{+}+S_{n+1,0}=b,\left(i-A_{n, 0}\right)^{+}+S_{n+1,0}=j\right\} .
\end{aligned}
$$


The equations also show that $\left(A_{n, 0}, S_{n+1,0}\right)$ is independent of $\left(A_{n-1,1}, J_{n}\right)$. Since the process $\left\{\left(A_{n, 1}, J_{n+1}\right)\right\}_{n \in \mathbb{Z}}$ is stationary, its marginal must be the unique invariant distribution of transition (7.22), namely

$P\left(A_{n-1,1}=k, J_{n}=j\right)=\left(1-\alpha^{-1}\right)^{k-1} \alpha^{-1} \cdot\left(1-f(\alpha)^{-1}\right)^{j-1} f(\alpha)^{-1} \quad$ for $k, j \in \mathbb{N}$,

with $f(\alpha)=m_{0} \frac{\alpha-1}{\alpha-m_{0}}$. This shows that i.i.d. mean $\alpha$ geometric is a queuing fixed point. Next solve for $\gamma(s)=\inf _{\alpha>m_{0}}\{\alpha s+f(\alpha)\}$. The unique minimizing $\alpha$ in terms of $s=\xi \cdot e_{1} / \xi \cdot e_{2}$ is $\alpha=m_{0}+\sigma \sqrt{\xi \cdot e_{2} / \xi \cdot e_{1}}$ which defines the bijection between $\xi \in$ ri $\mathscr{U}$ and $\alpha \in\left(m_{0}, \infty\right)$. From this

$$
f(\alpha)=m_{0} \frac{\alpha-1}{\alpha-m_{0}}=m_{0}+\sigma \sqrt{\xi \cdot e_{1} / \xi \cdot e_{2}} .
$$

Finding $\gamma(s)$ gives (3.8) via (4.1) and then $\gamma^{\prime}(s)$ gives (3.9) via (4.12) and (5.3).

The terms in the sum $J_{n}=S_{n, 0}+W_{n, 0}$ are independent, so we can also find the distribution of the waiting time:

$P\left(W_{n, 0}=0\right)=\frac{\alpha-m_{0}}{\alpha-1}, \quad P\left(W_{n, 0}=k\right)=\frac{m_{0}-1}{\alpha-1} \cdot\left(1-f(\alpha)^{-1}\right)^{k-1} f(\alpha)^{-1} \quad(k \geq 1)$.

Acknowledgements The authors thank Yuri Bakhtin and Michael Damron for useful discussions and two anonymous referees for valuable comments. N. Georgiou was partially supported by a Wylie postdoctoral fellowship at the University of Utah and the Strategic Development Fund (SDF) at the University of Sussex. F. Rassoul-Agha and N. Georgiou were partially supported by National Science Foundation grant DMS0747758. F. Rassoul-Agha was partially supported by National Science Foundation grant DMS-1407574 and by Simons Foundation grant 306576. T. Seppäläinen was partially supported by National Science Foundation grants DMS-1306777 and DMS-1602486, by Simons Foundation grant 338287, and by the Wisconsin Alumni Research Foundation.

\section{Appendix: Ergodic theorem for cocycles}

Cocycles satisfy a uniform ergodic theorem. The following is a special case of Theorem 9.3 of [28]. Note that a one-sided bound suffices for a hypothesis. Recall Definition 2.1 for the space $\mathscr{K}_{0}$ of centered cocycles.

Theorem 7.8 Assume $\mathbb{P}$ is ergodic under the transformations $\left\{T_{e_{i}}: i \in\{1,2\}\right\}$. Let $F \in \mathscr{K}_{0}$. Assume there exists a function $V$ such that for $\mathbb{P}$-a.e. $\omega$

$$
\varlimsup_{\varepsilon \searrow 0} \varlimsup_{n \rightarrow \infty} \max _{x:|x|_{1} \leq n} \frac{1}{n} \sum_{0 \leq k \leq \varepsilon n}\left|V\left(T_{x+k e_{i}} \omega\right)\right|=0 \quad \text { for } i \in\{1,2\}
$$

and $\max _{i \in\{1,2\}} F\left(\omega, 0, e_{i}\right) \leq V(\omega)$. Then

$$
\lim _{n \rightarrow \infty} \max _{\substack{x=z_{1}+\cdots+z_{n} \\ z_{1, n} \in\left\{e_{1}, e_{2}\right\}^{n}}} \frac{|F(\omega, 0, x)|}{n}=0 \quad \text { for } \mathbb{P} \text {-a.e. } \omega .
$$

If the process $\left\{V\left(T_{x} \omega\right): x \in \mathbb{Z}^{2}\right\}$ is i.i.d., then a sufficient condition for (7.23) is $\mathbb{E}\left(|V|^{p}\right)<\infty$ for some $p>2[50$, Lemma A.4]. 


\section{References}

1. Alm, S.E.: A note on a problem by Welsh in first-passage percolation. Comb. Probab. Comput. 7(1), $11-15(1998)$

2. Alm, S.E., Wierman, J.C.: Inequalities for means of restricted first-passage times in percolation theory. Comb. Probab. Comput. 8(4), 307-315 (1999). (Random graphs and combinatorial structures (Oberwolfach, 1997))

3. Armstrong, S.N., Souganidis, P.E.: Stochastic homogenization of Hamilton-Jacobi and degenerate Bellman equations in unbounded environments. J. Math. Pure Appl. (9) 97(5), 460-504 (2012)

4. Auffinger, A., Damron, M.: Differentiability at the edge of the percolation cone and related results in first-passage percolation. Probab. Theory Relat. Fields 156(1-2), 193-227 (2013)

5. Bakhtin, Y.: Burgers equation with random boundary conditions. Proc. Am. Math. Soc. 135(7), 22572262 (2007). (electronic)

6. Bakhtin, Y.: The Burgers equation with Poisson random forcing. Ann. Probab. 41(4), 2961-2989 (2013)

7. Bakhtin, Y.: Inviscid Burgers equation with random kick forcing in noncompact setting (2014). Preprint arXiv: 1406.5660

8. Bakhtin, Y., Cator, E., Khanin, K.: Space-time stationary solutions for the Burgers equation. J. Am. Math. Soc. 27(1), 193-238 (2014)

9. Bakhtin, Y., Khanin, K.: Localization and Perron-Frobenius theory for directed polymers. Mosc. Math. J. 10(4), 667-686, 838 (2010)

10. Balázs, M., Cator, E., Seppäläinen, T.: Cube root fluctuations for the corner growth model associated to the exclusion process. Electron. J. Probab. 11(42), 1094-1132 (2006). (electronic)

11. Burkholder, D.L.: Distribution function inequalities for martingales. Ann. Probab. 1, 19-42 (1973)

12. Carmona, P., Hu, Y.: On the partition function of a directed polymer in a Gaussian random environment. Probab. Theory Relat. Fields 124(3), 431-457 (2002)

13. Cator, E., Groeneboom, P.: Second class particles and cube root asymptotics for Hammersley's process. Ann. Probab. 34(4), 1273-1295 (2006)

14. Cator, E., Pimentel, L.P.R.: Busemann functions and equilibrium measures in last passage percolation models. Probab. Theory Relat. Fields 154(1-2), 89-125 (2012)

15. Cator, E., Pimentel, L.P.R.: Busemann functions and the speed of a second class particle in the rarefaction fan. Ann. Probab. 41(4), 2401-2425 (2013)

16. Cohn, H., Elkies, N., Propp, J.: Local statistics for random domino tilings of the Aztec diamond. Duke Math. J. 85(1), 117-166 (1996)

17. Comets, F., Shiga, T., Yoshida, N.: Directed polymers in a random environment: path localization and strong disorder. Bernoulli 9(4), 705-723 (2003)

18. Corwin, I.: The Kardar-Parisi-Zhang equation and universality class. Random Matrices Theory Appl. 1(1), 1130001, 76 (2012). doi:10.1142/S2010326311300014

19. Damron, M., Hanson, J.: Busemann functions and infinite geodesics in two-dimensional first-passage percolation. Commun. Math. Phys. 325(3), 917-963 (2014)

20. Durrett, R.: Oriented percolation in two dimensions. Ann. Probab. 12(4), 999-1040 (1984)

21. Durrett, R., Liggett, T.M.: The shape of the limit set in Richardson's growth model. Ann. Probab. 9(2), 186-193 (1981)

22. E, W., Khanin, K., Mazel, A., Sinai, Y.: Invariant measures for Burgers equation with stochastic forcing. Ann. Math (2) 151(3), 877-960 (2000)

23. Ferrari, P.A., Martin, J.B., Pimentel, L.P.R.: A phase transition for competition interfaces. Ann. Appl. Probab. 19(1), 281-317 (2009)

24. Ferrari, P.A., Pimentel, L.P.R.: Competition interfaces and second class particles. Ann. Probab. 33(4), 1235-1254 (2005)

25. Georgiou, N., Rassoul-Agha, F., Seppäläinen, T.: Stationary cocycles for the corner growth model (2014). Preprint arXiv:1404.7786

26. Georgiou, N., Rassoul-Agha, F., Seppäläinen, T.: Geodesics and the competition interface for the corner growth model. Probab. Theory Relat. Fields (2016). doi:10.1007/s00440-016-0734-0

27. Georgiou, N., Rassoul-Agha, F., Seppäläinen, T.: Variational formulas and cocycle solutions for directed polymer and percolation models. Commun. Math. Phys. (2016). To appear (arXiv:1311.0316)

28. Georgiou, N., Rassoul-Agha, F., Seppäläinen, T., Yılmaz, A.: Ratios of partition functions for the log-gamma polymer. Ann. Probab. 43(5), 2282-2331 (2015) 
29. Glynn, P.W., Whitt, W.: Departures from many queues in series. Ann. Appl. Probab. 1(4), 546-572 (1991)

30. Hoang, V.H., Khanin, K.: Random Burgers equation and Lagrangian systems in non-compact domains. Nonlinearity 16(3), 819-842 (2003)

31. Hoffman, C.: Coexistence for Richardson type competing spatial growth models. Ann. Appl. Probab. 15(1B), 739-747 (2005)

32. Hoffman, C.: Geodesics in first passage percolation. Ann. Appl. Probab. 18(5), 1944-1969 (2008)

33. den Hollander, F.: Random polymers. Lecture Notes in Mathematics, vol. 1974. Springer-Verlag, Berlin (2009)

34. Howard, C.D., Newman, C.M.: Geodesics and spanning trees for Euclidean first-passage percolation. Ann. Probab. 29(2), 577-623 (2001)

35. Iturriaga, R., Khanin, K.: Burgers turbulence and random Lagrangian systems. Commun. Math. Phys. 232(3), 377-428 (2003)

36. Jockusch, W., Propp, J., Shor, P.: Random domino tilings and the arctic circle theorem (1998). arXiv:math/9801068

37. Johansson, K.: Shape fluctuations and random matrices. Commun. Math. Phys. 209(2), 437-476(2000)

38. Johansson, K.: Random matrices and determinantal processes. In: Mathematical statistical physics, pp. 1-55. Elsevier B. V., Amsterdam (2006)

39. Licea, C., Newman, C.M.: Geodesics in two-dimensional first-passage percolation. Ann. Probab. 24(1), 399-410 (1996)

40. Loynes, R.M.: The stability of a queue with non-independent interarrival and service times. Proc. Camb. Philos. Soc. 58, 497-520 (1962)

41. Mairesse, J., Prabhakar, B.: The existence of fixed points for the ·/GI/1 queue. Ann. Probab. 31(4), 2216-2236 (2003)

42. Marchand, R.: Strict inequalities for the time constant in first passage percolation. Ann. Appl. Probab. 12(3), 1001-1038 (2002)

43. Martin, J.B.: Limiting shape for directed percolation models. Ann. Probab. 32(4), 2908-2937 (2004)

44. Muth, E.J.: The reversibility property of production lines. Management Sci. 25(2), 152-158 (1979/80)

45. Newman, C.M.: A surface view of first-passage percolation. In: Proceedings of the international congress of mathematicians, Vol. 1, 2 (Zürich, 1994), pp. 1017-1023. Birkhäuser, Basel (1995)

46. Pimentel, L.P.R.: Multitype shape theorems for first passage percolation models. Adv. Appl. Probab. 39(1), 53-76 (2007)

47. Pimentel, L.P.R.: Duality between coalescence times and exit points in last-passage percolation models. Ann. Probab. (2015). To appear (arXiv:1307.7769)

48. Prabhakar, B.: The attractiveness of the fixed points of a $/ / G I / 1$ queue. Ann. Probab. 31(4), 2237-2269 (2003)

49. Rassoul-Agha, F., Seppäläinen, T.: Quenched point-to-point free energy for random walks in random potentials. Probab. Theory Relat. Fields 158(3-4), 711-750 (2014)

50. Rassoul-Agha, F., Seppäläinen, T., Yılmaz, A.: Quenched free energy and large deviations for random walks in random potentials. Commun. Pure Appl. Math. 66(2), 202-244 (2013)

51. Rassoul-Agha, F., Seppäläinen, T., Yılmaz, A.: Variational formulas and disorder regimes of random walks in random potentials. Bernoulli (2016). To appear (arXiv:1410.4474)

52. Rost, H.: Nonequilibrium behaviour of a many particle process: density profile and local equilibria. Z. Wahrsch. Verw. Gebiete 58(1), 41-53 (1981)

53. Seppäläinen, T.: Coupling the totally asymmetric simple exclusion process with a moving interface. Markov Process. Relat. Fields 4(4), 593-628 (1998). (I Brazilian School in Probability (Rio de Janeiro, 1997))

54. Seppäläinen, T.: Hydrodynamic scaling, convex duality and asymptotic shapes of growth models. Markov Process. Relat. Fields 4(1), 1-26 (1998)

55. Seppäläinen, T.: Scaling for a one-dimensional directed polymer with boundary conditions. Ann. Probab. 40(1), 19-73 (2012). Corrected version available at arXiv:0911.2446

56. Wüthrich, M.V.: Asymptotic behaviour of semi-infinite geodesics for maximal increasing subsequences in the plane. In: In and out of equilibrium (Mambucaba, 2000), Progr. Probab., vol. 51, pp. 205-226. Birkhäuser Boston, Boston (2002) 